\title{
INSTANTANEOUS CENTER OF ROTATION SHIFTS IN SYMPTOMATIC ANTERIOR CRUCIATE LIGAMENT DEFICIENT KNEE JOINTS
}

\author{
by
}

Michael John Simmonds $\subset$

A Thesis Submitted to the Faculty of Graduate Studies and Research in Partial Fulfilment of the Requirements for the Degree of Masters of Arts (Education)

Department of Physicai Education

Division of Graduate Studies and Research

Faculty of Education

McGill University

Montreal, Quebec

May, 1990 


\section{Acknowledgements}

This thesis could not have been completed without the direct influence of some very special people. I am forever grateful to Monica Kosiuk and Sonya Matthews whose encouraging comments and constant assistance contributed significantly to the development of this project at all stages of its evolution. Their continual emotional support and commitment to friendship enabled me to persevere through some difficult times. Thanks to Dr. Eric Leczner for his clinical input and constant referral of suhjects to the lab. Thanks to Vassilis Vardaxis whose computer prowess was instrumental in completing the analysis part of the investigation. My parents (Bob and Mary) and siblings (Paul and Kelli) also deserve recognition. Not only did they support my decision to pursue graduate school but they also bolstered my resolve to complete the task. Finally I wish to express my sincere gratitude and appreciaition in Dr. Blainc Hoshizaki whose patience, guidance, and influence instilled the attitudes necessary to finish this undertaking. His insightful nature and personal philosophies will continue to play an important role in my life, as they have throughout the duration of this work. 


\begin{abstract}
The purpose of this investigation was to document the influence of the anterior cruciate ligament (ACL) in controlling the Hynamic interaction between the femur and tibia. Twenty subjects were initially selected to participate based on the results of arthroscopic and proprioceptive tests which established the presence of a uni-lateral, third degree rupture of the ACL. Examination of the contralateral knee confirmed the absence of previous ligament disruption. The characteristics of the deficiency were quantified on the Genucom Knee Arthrometer (GKA). These measures documented the extent of bilateral anterior laxity asymmetry that existed for each subject. Sagittal laxity asymmetries of $5 \mathrm{~mm}$ or greater served as an inclusion criteria for protocol completion and reduced the sample from twenty subjects to fifteen. Rotation shifts of the instant center knee axis were documented in two dimensions to reflect the effects of in-vivo cartilage degeneration. Three anatomical points were identified on the leg with the subjects' thigh securely positioned in the GKA. These included: (1) the tibial tuberosity, (2) a point on the tibial crest approximately $15 \mathrm{~cm}$ inferior to it, and (3) the medial epicondyle of the femur. A BASELINE (tm) goniometer was attached to the leg and monitored flexion angles. $A$ $110 \pm 5 \mathrm{~N}$ force was applied at the joint throughout a range of motion (ROM) beginning at $100^{\circ}$ and continuing to $-10^{\circ}$ by $10^{\circ}$ increments. The anatomical markers were digitized throughout the range and quantified in reference to three orthogonal planes: (1) frontal, (2) sagittal, and (3) transverse. Numerical coordinates generated in the sagittal plane were used to compute the instant center (IC) of the knee according to the method of Reuleaux (1876). A spline function established the best fitting curve of the IC coordinates obtained throughout the ROM. Displacement variables were extrapolated from joint rotation profiles generated from these coordinates. Abnormal displacement migrations of the IC parameter were demonstrated to occur in extension. Migration displacements evaluated between $30^{\circ}$ and $0^{\circ}$ shifted anteriorly $5.07 \pm 1.86 \mathrm{~mm}$ for intact knees and $6.97 \pm 2.06 \mathrm{~mm}$ for involved knees. This was determined io be significant at the 0.05 level of confidence and correlated well with clinical findings. Mean migration displacements evaluated in flexion (between $100^{\circ}$ and $70^{\circ}$ ) were not found to differ significantly between knee conditions.
\end{abstract}




\begin{abstract}
Abstrait
Le but de cette étude était de documenter l'influence du ligament croissé antérieur en déterminant l'interaction dynamique entre le fémur et le tibia. Le première sélection de vingts participants était basée sur les résultats de deux examens. Les examens arthroscopic et proprioception ont établi la présence d'une rupture unilatéral du ligament croissé antérieur. Les caractéristiques de la rupture ont été mesuré par la GENUCOM. Le mesure de déplacement antérieur du tibia en relation du fémur à établi le degré de non-symétrie entre les deux genoux de chaque participants. Une différence de $5 \mathrm{~mm}$ ou plus d'élévarion pour le déplacement antérieur entre les deux genoux a servi comme un critère d'inclusion qui, en effet, a réduit le nombre de participants de vingts personnes à quinze. Les changements de rotation pour le centre instantané de gennu ont été documenté en deux dimensions pour refléter les effets d'une dé_inérescence du cartilage. Avec la cuisse du suject bien sécurer dans la GENUCOM, trois points anatomic ont été identifiés sur la jambe: (1) frontal, (2) un point sur la crête du tibia $15 \mathrm{~cm}$ en bas de la tuberosité et (3) l'épicondyle medial du fémur. Une goniomèter était attaché aux jambes pour mesurer les angles du flexion. Une force de $110 \pm 5 \mathrm{~N}$ était appliquée à l'articulation du genou à un angle de $10^{\circ}$ jusqu'à $100^{\circ}$ par une augmentation de $10^{\circ}$ chaque fois. Les coordonnées à trois dimensions ont décrit les points anatomic en trois plan: (1) frontal, (2) sagittal, et (3) transverse. Les coordonnées générer dans le plan sagittal ont été utilisé pour calculer le centre instantané du genou par la méthode Reuleaux (1876). Une fonction spline a été utilisé pour produire une courbe qui représentait les coordonnées du centre instantané à chaque angle de flexion. Les variables du déplacement pour la rotation de l'articulation on été extrapolé. Les résultats ont démontré des migrations exceptionnelles du centre instantané quand l'articulation du genou était en extension. Entre $30^{\circ}$ et $0^{\circ}$ de flexion le déplacement antérieur pour les genoux non-blessés était $5.07 \pm 1.86 \mathrm{~mm}$, par contre le déplacement était $6.97 \pm 2.06 \mathrm{~mm}$ pour les genoux blessés. Cette différence était significatif à $\mathrm{a}=0.05$ et en même temps était en corrélation avec les résultats clinique. Il n'y avait pas de différence significatif pour les migrations qui mesuraient entre $100^{\circ}$ et $70^{\circ}$ de flexion.
\end{abstract}




\section{CHAPTER I}

\section{INTRODUCTION}

1.1 Nature and Scope of the Problem .................................... 2

1.2 Statement of the Problem ............................................. 5

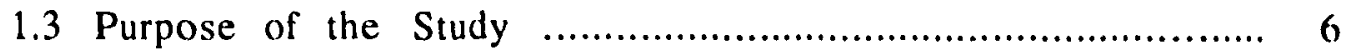

1.4 Secondary Research Hypotheses ..................................... 7

1.5 Primary Research Hypotheses .......................................... 8

1.6 Delimitations and Limitations ..................................... 8

1.7 Abbreviations ........................................................... 9

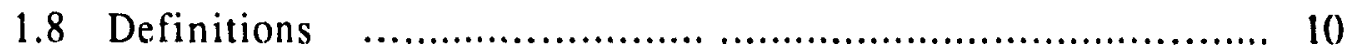

\section{CHAPTER II}

\section{REVIEW OF LITERATURE}

2.1 Introduction ........................................................ 11

2.2 Mechanism of Injury ............................................ 12

2.3 Anterior Cruciate Ligament Anatomy ............................... 13

2.4 Knee Joint Geometry ............................................... 14

2.5 Knee Joint Kinematics ............................................. 15

2.6 The Instant Center Pathway ....................................... 16

2.7 The Effect of ACL Insufficiency ................................... 18

2.8 Passive Structures ............................................... 22

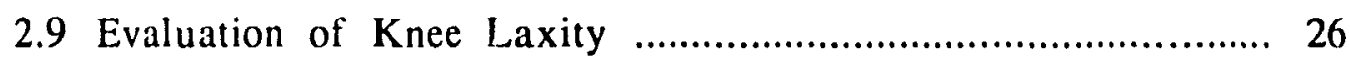

2.9.1 The Neutral Point ...................................................... 27

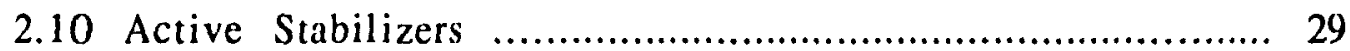

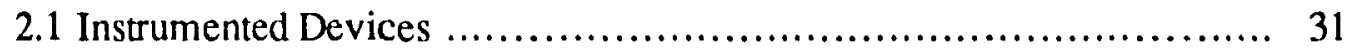

2.11.1 The Genucom Knee Analyzer .............................. 32

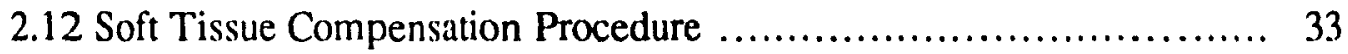

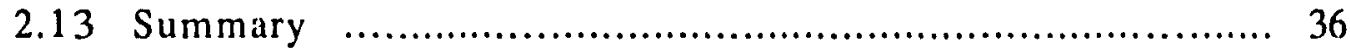




\section{METHODS AND PROCEDURES}

3.1 Introduction ...................................................... 38

3.2 Subject Selection ...................................................... 39

3.3 Description of the Study ............................................ 40

3.3.1 Part I ........................................................ 40

3.3.2 Part II ........................................................ 42

3.4 The Genucom Test ................................................ 43

3.4.1 Testing Procedure ........................................ 46

3.5 Experimental Design .............................................. 48

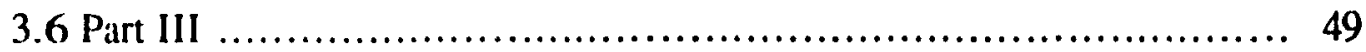

3.7 Calculation of Instant Center ..................................... 53

3.8 Statistical Analysis .................................................. 54

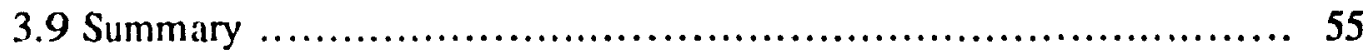

\section{CIIAPTER IV}

\section{RESULTS}

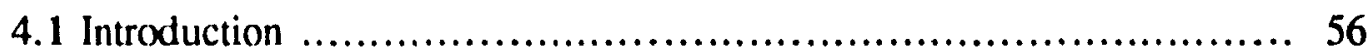

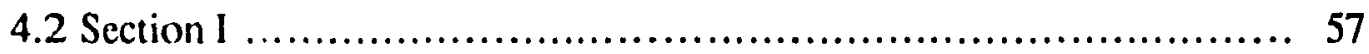

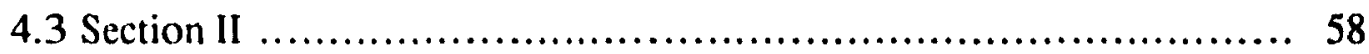

4.3.1 Subject Information ....................................... 58

4.3.2 One-Way ANOVA Results ................................. 60

4.3.3 Descriptive Statistics ....................................... 62

4.3.4 Two-Factor ANOVA Summary Table ........................... 64

4.3.5 Tukey Post-Hoc Mean Comparisons ................................... 65

4.4 Section III........................................................ 66

4.4.1 Intra-Subject Shape Reliability ......................................... 66

4.4.2 One-Way ANOVA Results ................................ 67

4.4.3 Descriptive Statistics .......................................... 68

4.4.4 Displacement Piofile Assessment ............................. 69 


\section{DISCUSSION OF RESULTS}

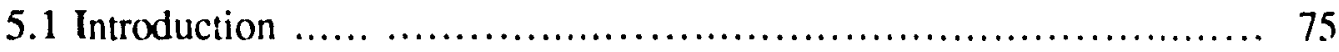

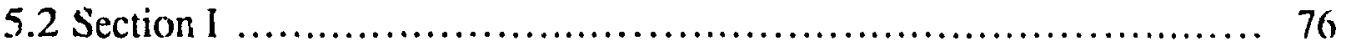

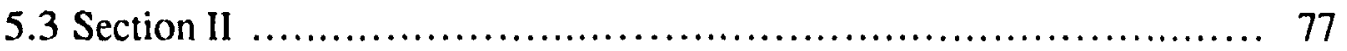

5.3 .1 Laxity Measures ............................................ 77

5.3.2 Stiffness Measures ............................................. 80

5.3.3 Torsional Instabilities ....................................... 83

5.4 Section III ........................................................... 83

5.4.1 Intra-Subject Reliability of IC Profiles ............................. 84

5.4.2 Displacement Profile Assessment ............................... 85

5.4 .3 Clinical Implications ........................................... 88

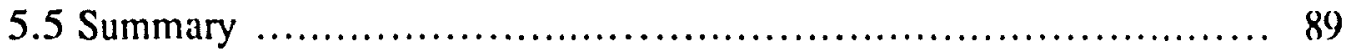

\section{CHAPTER VI}

\section{SUMMARY AND CONCLUSIONS}

6.1 Introduction ......................................................... 91

6.2 Results and Findings ....................................................... 91

6.2.1 Three Dimensional Motion Characteristics ......................... 91

6.2.2 Profile Assessment of the IC of Rotation ............................. 93

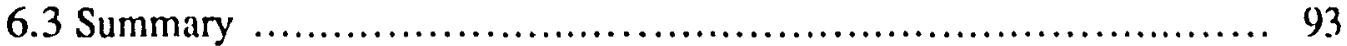

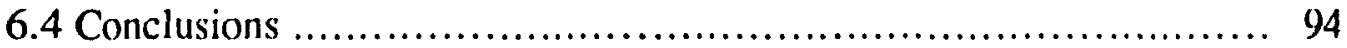

6.5 Recommendations for Further Study ............................... 95

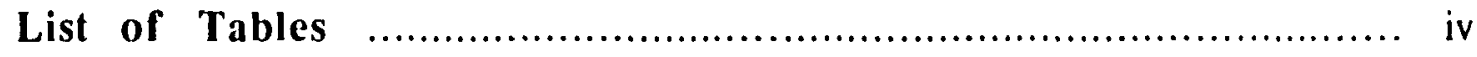

List of Figures ................................................................

List of Appendices ......................................................... vi

References...................................................................... 96-101

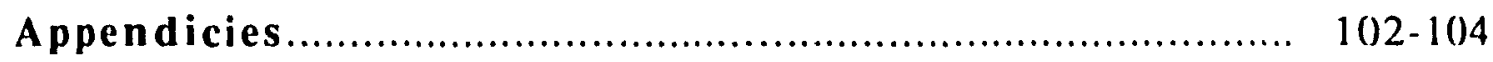




\section{LIST OF TABLES}

Table

Page

$1 \wedge \quad$ Shape Reliability of IC of Rotation Profiles .......................... 57

1B IC of Rotation Variabilty; Single Subject ........................... 57

2 Subject Information Summary ........................................ 59

3A-B One-Way ANOVA Summaries of Motion Parameters ............ 60

3C-F One-Way ANOVA Summaries of Motion Parameters ............. 61

3G-II One-Way ANOVA Summaries of Motion Parameters ............ 62

4A-B Descriptive Statistics of Motion Parameters ......................... 63

4C-E Descriptive Statistics of Motion Parameters .......................... 64

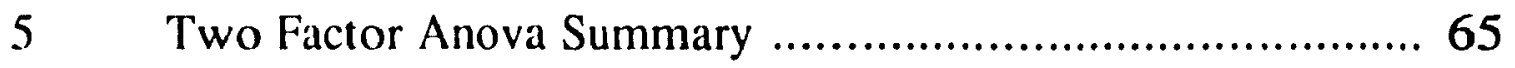

6 Tukey Post-Hoc Mean Comparisons …................................. 65

$7 \quad$ Intra-Subject Reliability Data ......................................... 67

8-9 One-Way ANOVA Summaries of IC of Rotation .................... 68

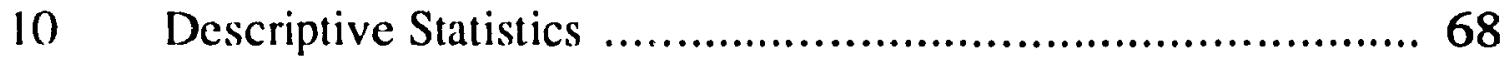




\section{LIST OF FIGURES}

Figure

Page

1A Tension Changes of ACL Band Components in Extension........... 20

1B Tension Changes of ACL Band Components in Flexion ............. 20

2 Directional Áxes of GKA …………....................................... 34

3 Anatomical Landmarkers Used to Compute Knee Center ............ 45

4 Schematic of Loading Mechanism .......................................... 52

5 IC Profile of an Involved Knee Along a Vertical Axis ................. 70

6 IC Profile of an Intact knee Along a Vertical Axis .................... 71

7 IC Profile of an Involved Knee Along a Sagittal Axis ................. 73

8 IC Profile of an Intact Knee Along a Sagittal Axis .................... 74

9 Roller - Trough Mechanism in Flexion ...................................... 79

10 Roller - Trough Mechanism in Extension ................................... 81 


\section{LIST OF APPENDICES}

$\begin{array}{ll}\text { Appendix } & \text { Page }\end{array}$

A Clinical Grading Scale ............................................. 102

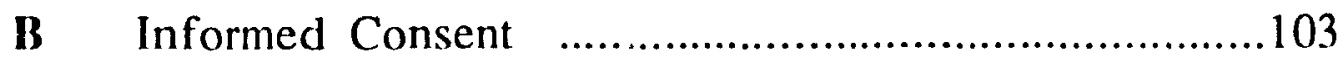

C Patient Information Summary …………………...........104 


\section{CHAPTER I}

\section{INTRODUCTION}

Numerous attempts by researchers and clinicians to describe the mechanics of the knee joint abound in the literature. Despite the specificity of clinical evaluations, technological advancements in testing apparatus, complex mathematical modeling, and the sophisticated power of computers, an accurate description of knee motion (and related kinematic parameters) still presents unique problems to the researcher. In part, this problem stems from the complexity of the knee joint itself; the accepted idcology among scientists and clinicians that knee motion is functionally three dimensional in nature (Kennedy et al., i974; Markolf et al., 1976, Noyes et al., 1980). However, uni-planar analyses are commonly performed by researchers in

an attempt to document knee motion in specific terms. Although this approach seemingly negates the true physiological structure of the joint, the assumption is an acceptable one because knee motion occurs primarily in one plane; the sagittal plane (Frankel \& Nordin, 1980).

In an attempt to describe the influence of the anterior cruciate ligament $(\mathrm{ACL})$ in controlling knee joint mechanics, a methodology is proposed which will objectively quantify criterion variables influenced by the geometrical configuration of the joint surface and the compliant properties of the supporting ligaments. In this way uni and multi-planar motion parameters will be obtained to specifically document the effects of ACL dysfunction on joint integrity. 


\section{NATURE AND SCOPE OF THE PROBLEM}

In the past, the response of developing adequate methodologies for evaluating knee joint stability have been rooted in an attempt to determine the roles of various ligamentous structures in-vitro. Markolf et al., (1976) measured the force-displacement response curves of thirty-five normal cadaver knees with the intention of quantifying knee joint stability. The non-linear load-displacement curves demonstrated that laxity and stiffness characteristics reflected the structural integrity of the joint. Although isolated section of the ACL produced the greatest measure in anterior laxity, stability was affected to some extent by virtually every sectioning procedure.

Other studies have examined the translational and rotational characteristics of the loaded tibia relative to a fixed femur. Marquette (1988) suggested the most problematic and destructive occurrence to the ACL deficient patient was the resulting rotatory instability that often accompanied its rupture. Lipke et al.. (1981) examined the anterolateral and anteromedial stability of seventeen fresh cadaver knees. He observed statistically significant increases in anterolateral instability when the ACL was sectioned. This confirmed the clinical $i$ 'npression of others that the $\Lambda \mathrm{CL}$ appeared to be the primary constraint preventing excessive rotation of the tibia on the femur. A similar study was conducted by Shoemaker \& Markolf (1985). An apparatus was developed to simulate compressive loads on the knee joint without restricting other motions of the knee. In this way the force-displacement and tibial torque rotation responses of fresh cadaver specimens could be examined. The response curves were analyzed in terms 
of stiffness and laxity parameters. It was revealed that sectioning of the ACL always increased anterior and torsional laxity while eliciting corresponding reductions in stiffness.

These in-vitro studies are significant for two reasons. Foremost, they have established the relationship between laxity and stiffness parameters with in-vivo values obtained in normal subjects. Such comparisons are essential to ensure that the stiffness and laxity parameters which are measured accurately reflect the status of the knee joint and not artifacts produced by various testing apparatus (Markolf et al., 1984). Secondly, such investigations highlight the complex interaction that exists between anterior displacement and tibial rotation. This complexity stems in part from the the compliant properties of the ACL and in part from the geometrical configuration of articulating bone.

Internal derangements of the knee can also be determined by the instant center (IC) pathway of the joint (Soudan \& Van Audekercke, 1979; Frankel et al., 1971; Frankel \& Nordin, 1980). Since the instant center pathway of the joint describes joint movement, and since joint movement correlates with anatomical restrictions, it follows that the place and pathway of the instant center will reflect the structural integrity of the joint. Frankel et al., (1971) described a kinematic method to elucidate the correlation that existed between tissue derangement and mechanical derangement in two dimensions. The instant center pathway was determined by the method of Reuleaux (1876) in which the displacement of two points on a link were identified in successive position:s. Perpendicular bisectors were then drawn for each pair of displaced points, 
the intersection of which located the centroid for that particular displacement. In this way the centroids were plotted and comparisons were macis between intact and involved knees. Results of the investigation revealed abnormal instant center pathways for the involved knee when compared to consistent pathways that were observed for the twenty-five intact knees (Frankel et al., 1971).

Gerber \& Matter (1983) confirmed this observation and reported that degenerative changes in the structural integrity of the knee joint were ultimately reflected by discrepancies in instant center profiles between involved and intact knees. He suggested that ligament deficiency resulted in a breakdown of some "normal" kinematic synergy between the femur and tibia. Müeller (1985) documented the nature of this synergy. He suggested a basic mechanism of movement between the femur and tibia could be described as being a combination of "rolling" and "gliding". This mechanism was directly influenced by the architecture of the joint surface. Müeller hypothesized that if "pure" rolling were to occur the femur would literally "roll off" the tibial plateau at approximately $45^{\circ}$ flexion. Similarly, if "pure" gliding were to occur the metaphysis of the femur would engage on the posterior rim of the tibial plateau at approximately $130^{\circ}$ of flexion. Furthermore, he discovered an accurate plot of the contact points between the femur and tibia revealed that a normal rolling to gliding ratio did not remain constant throughout the entire range of motion. In actuality, consistent synergistic ratios were discovered to be "range specific" occurring in extension and late flexion only. 


\subsection{STATEMENT OF THE PROBLEM}

The literature is replete with evidence documenting the functional importance of the ACL in promoting knee joint stability. This stems from the results of clinical investigations that indicate the ACL is the most frequently torn ligament within the knee joint (Johnson, 1983). However, despite the frequency of injury to this particular structure, physicians and researchers have yet to develop an accurate, non-invasive methodology of quantifying a variety of motion parameters that reflect actual degenerative changes in joint mechanics that accompany ligament dysfunction. Typically physicians rely on clinical techniques to document joint deficiencies. The examiner applies unknown forces and moments to the joint and subsequently observes the displacements and rotations he elicits. This becomes problematic when discrepant diagnoses result from the subjective interpretation of the clinical findings.

Clearly such proprioceptive techniques for assessing knee joint stability are inadequate. The ACL is the only completely intra-articular ligament of the knee that has no capsular attachments. This precludes the possibility of palpating for structural deficiencies (Odensten \& Gilquist, 1985). Although many experienced clinicians develop a qualitative "feel" for the laxity and stiffness parameters elucidated by joint manipulation, the reliability of the examining procedure is still subjective and questionable (Markolf et al., 1976; Markolf \& Amstutz, 1987; Marshall \& Baugher, 1980). 
In an attempt to identify a more effective approach of characterizing knee joint dysfunction, it is imperative to adopt a mechanical measure of relative tibio-femoral motion for two reasons. Foremost, it would provide objective data to assist in decision making regarding knee joint stability. Secondly, it would provide information regarding the relative effectiveness of rehabilitative strategies. Ideally such measures should reflect the three dimensional characteristics of the knee joint. However, kinematic descriptions that attempt to describe joint synergy frequently adopt a uni-planar model of knee motion. Both methods can yield useful biomechanical data (Frankel \& Nordin, 1980). Regardless of the approach taken, researchers agree that the parameters must reflect the geometrical configuration of the joint surfaces and the compliant properties of the ligaments, if they are to be considered useful descriptors of knee motion. Motion parameters sensitive to these underlying constraints will most accurately reflect the unique structural interaction which exists between bone and supporting compliant ligaments. Discrepancies resulting from a breakdown in this interaction will therefore be reflected by contralateral differences between dependant measures obtained bilateraly for uni-lateral deficient subjects. In this way quantifiable phenomenon resulting from structural deformation to the ACL can objectively be quantified.

\subsection{PURPOSE OF THE STUDY}

The purpose of this investigation was to employ a knee arthrometer as an objective testing device to characterize instant center joint rotation patterns of ACL deficient knee joints under a loaded condition and throughout a 
range of motion. Displacement shifts of this parameter are thought to reflect the dynamic relationship between bone and compliant ligaments (Gerber \& Matter, 1983; Moeinzadeh et al., 1983). A methodology was developed to document this inter-active synergy. This facilitated the need to adopt a protocol which quantified three dimensional and uni-planar motion parameters in specific terms.

Uni-planar motion parameters established quantifiable laxity and stiffness characteristics for each subject. The results of these measures provided objective documentation from which to select subjects exhibiting "critcriaestablished" joint symptoms. Clinical techniques elicited the motion parameters of interest. These parameters were defined and tested in this study as secondary research hypotheses. This reflected the need for establishing the structural integrity of the joint as a condition for subject inclusion (according to an established laxity asymmetry criteria) while at the same time, demonstrated the subordinate nature of these hypotheses in light of the primary research hypotheses; the focus of the investigation.

\subsection{SECONDARY RESEARCH HYPOTHESES}

(1) There will be no significant difference between anterior terminal laxity (AL) parameters evaluated at $130 \pm 5$ Newtons by the Lachmans test performed at $30^{\circ}$ and $20^{\circ}$ flexion for intact and involved knees.

(2) There will be no significant difference between anterior midrange stiffness (AMRS) parameters evaluated between 40 and 60 Newtons while 
performing the Lachmans test at $30^{\circ}$ and $20^{\circ}$ flexion for intact and involved knees.

(3) There will be no significant difference between internal tibial rotation (ITR) parameters measured in degrees at $9 \pm 2 \mathrm{~N}$-m while performing tibial torsion tests at $90^{\circ}$ flexion for intact and involved knees.

(4) There will be no significant difference between anterior translation parameters of the lateral tibial plateau (ATLP) measured in millimetres while performing internal tibial torsion tests at $90^{\circ}$ flexion for intact and involved knees.

\subsection{PRIMARY RESEARCH HYPOTHESES}

(1) There will be no significant differences between displacement parameters of the IC of rotation measured in the sagittal plane for intact and involved knees between $30^{\circ}$ and $0^{\circ}$ flexion in the vertical $(\mathrm{Y})$ axis.

(2) There will be no significant differences between displacement parameters of the IC of rotation measured in the sagittal plane for intact and involved knees between $100^{\circ}$ and $70^{\circ}$ flexion in the sagittal $(\mathrm{Z})$ axis.

\subsection{DELIMITATIONS AND LIMUTATIONS}

The following delimitations and limitations apply to this study: 


\section{Delimitations}

(1) Subject profile is limited to ACL deficient knees.

\section{Limitations}

(1) Asymmetrical differences between right and left knees prior to the subjects injury are assumed to be statistically negligible.

(2) Subjects included in this investigation may not have isolated ACL tears due to the likelihood of damage to other supporting structures upon injury.

(3) The active state of the muscles involved in contracting against a resistive load was not directly monitored.

(4) Instant center motion parameters are quantified in the sagittal plane on the assumption of uni-planar motion.

\subsection{ABBREVIATIONS}

(1) AMB - anteromedial band; a band of fassicles originating at the proximal aspect of the femur attachment and inserting at the anteromedial aspect of the tibial attachment.

(2) PLB - posterolateral band; the remaining band of fassicles at the posterolateral aspect of the tibial attachment. 
(3) ATLP - anterior translation of the lateral tibial plateau; subluxation of the lateral tibial plateau relative to a fixed femur.

(4) GKA - Genucom Knee Analyzer; a knee arthrometer developed to measure three dimensional motion parameters reflecting knee joint dysfunction.

(5) AL - anterior terminal laxity; a measure of the amount of anterior tibial translation in millimetres of the tibia relative to a fixed femur in response to an applied anterior load of $130 \pm 5$ Newtons.

(6) AMRS - anterior midrange stiffness; a measure of ligament compliance measured in $\mathrm{N} / \mathrm{mm}$. It is computed as the inverse slope of forcedisplacement curves obtained by the GKA.

\subsection{Definitions}

(1) Anterior cruciate ligament deficiency is defined in this study as a condition in which subjects exhibit bilateral anterior laxity asymmetries of five millimetres or greater in the sagittal plane. These subjects were identified by clinical tests performed on the GKA. All subjects were initially referred to the study by an Orthopaedic Surgeon who had originally diagnosed their deficiency by proprioceptive and arthroscopic means. 


\section{CHAP'TER II}

\section{REVIEW OF THE LITERATURE}

\subsection{INTRODUCTION}

The knee joint is the largest and perhaps the most complex joint in the human body. More accurately it is commonly described as being an "interactive network" between passive and active structures that combine to promote knee joint stability. Passive structures include the geometry of articulating bone and supporting ligaments while the active structures constitute supporting musculature and tendons (McLeod, 1985). Together these structures interface to effect joint synergy and reflect the integrity of joint kinematics. Ultimately, this interaction resilts in a variety of unique three dimensional movements which occur at the joint. In directional terms these movements include flexion, extension, translation, and rotation.

However, the knee is commonly studied as a two-dimensional, uni-planar "hinge" joint for two reasons. Foremost, by restricting knee motion to uniplanar measures it becomes possible to focus upon the essentials of the problem under investigation (Moinzadeh et al., 1983). Ultimately such studies establish the foundation for the development of more realistic three dimensional models that attempt to describe knee motion. Secondly, the role of the $\mathrm{ACL}$ in promoting joint integrity is well documented. The general consensus among researchers is the belief that the ACL is a primary anterior and secondary rotatory stabilizer of the knee (Markolf et 
al., 1976; Kennedy et al., 1974; Noyes et al., 1980). Rovere \& Adair (1983) reported that the ACL provided an average of $86 \%$ of the total resisting force to anterior translation of the tibia. Müeller et al., (1988) declared that the function of knee measurements was to reflect motion parameters influenced by the greatest possible motion between the tibia and femur. Since the ACL is the primary restraint of anterior translation, and since the bulk of knee motion occurs primarily in the the sagittal plane, it is evident that two-dimensional measures can yield legitimate, kinematic data in the description of joint motion.

\subsection{MECHANISM OF INJURY}

There is little doubt that the ACL is the most frequently torn ligament within the knee joint (Johnson, 1983). Although Noyes et al., (1983) suggested football and basketball related mishaps constituted the majority of ACL ruptures in athletes, virtually all sporting activities can potentially subject the ligament to a similar fate. This may be attributed to the mechanism by which structural deformation is typically associated. Three major categories of injury have been proposed in the literature: (1) forced hyper-extension, (2) forced flexion and external rotation of the tibia on the femur, and (3) forced hyper-flexion of the knee (King et al., 1986). Kennedy et al., (1974) suggested the most common mechanism included some component of hyper-extension with or withcut tibial rotation. In fact the "isolated rupture" scenario for ACL's was characterized by King et al., (1986) as occurring in deceleration activities in which the foot was firmly planted, the knec extended, and the tibia internally rotated. Another mechanism commonly cited as eliciting ACL damage are anteriorly 
directed forces applied to the posterior aspect of the proximal tibia (King et al., 1986). This mechanism occurred with the knee positioned in complete flexion or extension and typically resulted from a "clipping type" action commonly witnessed in American football.

\subsection{ANTERIOR CRUCIATE LIGAMENT ANATOMY}

The ACL is an intra-articular but extra-synovial ligament. As such, it lies within the capsule of the joint but not within the synovial membrane (Welsh, 1980). The dimensions of the ACL have been examined in numerous investigations. Its average length was found to be $38.2 \pm 6.8$ $\mathrm{mm}$; its average width $11.1 \pm 3.9 \mathrm{~mm}$ (Rovere \& Adair, 1983). It attaches to the anterior aspect of the tibial plateau in front of the tibial spine and extends upwards and backward through the supracondylar notch to insert over the back of the lateral femoral condyle. As the ligament passes backward it "turns on itself" through $90^{\circ}$ and inserts (not as a distinct cord) but rather spreads out over a broad flattened area. Functionally this results in portions of the ligament remaining under tension and accounts for the ability of the ACL to stabilize the joint throughout a range of motion (Welsh, 1980; Girgis et al., 1975). Furthermore, the "turning" of the ligament on itself gives the ACL an "isometric" characteristic such that no actual lengthening or shortening of the ligament occurs with movement. More accurately, there is is a tightening of different components within the ligament throughout different phases of movement. The actual overall length of the ACL remains unaltered (Welsh, 1980). This unique structural feature has clinical importance. It accounts for the fact that the $\mathrm{ACL}$ is 
commonly torn as an isolated entity from relatively inconsequential trauma (Welsh, 1980; Kennedy et al.,1974).

\subsection{KNEE JOINT GEOMETRY}

The unequal profile lengths of the femoral condyles has been described even in historical writings. Viewing the femur axially from below, one is struck by its asymmetry. In general, the medial condyle is distinguished by the presence of an extra weight bearing surface (Müeller, 1983). The tibial plateaus provide reciprocal articular surfaces for the femoral condyles with the medial tibial plateau being slightly concave while the lateral plateau presents a "cartilage cap" making it slightly convex in profile (Welsh, 1980). The unique structural features of these bony surfaces combine with the compliant properties of ligaments to facilitate a variety of three dimensional movements throughout the normal range of motion. These include flexion, extension, translation, and rotation.

Müeller (1985) described automatic rotation which he observed to begin in the last $20^{\circ}$ of extension. During the final stage of extension, the lateral femoral condyle "impinged" on the tibia at the terminal sulcus, while the annular sector of the medial condyle was thus freed to glide backwards. This was a direct result of the longer, more curved articular surface of the medial femoral condyle. Lateral rotation of the tibia on the femur initiated the "screw-home" mechanism of the joint. This phenomenon characterized the extreme complexity of knee joint dynamics and has provoked many controversial functions of the ACL to be proposed. The general consensus now appears to be that rotational stability is a function of the ACL 
secondary to its primary role of resisting anterior displacement of the tibia on the femur in the sagittal plane (Butler et al., 1980; McLeod, 1985; Gurtler et al., 1987).

\subsection{KNEE LOINT KINEMATICS}

It is generally agreed that the true physiological motion at the knee is a combination of rolling and gliding between the tibia and the femur (Frankel et al., 1971; Müeller, 1985). Many different authors have studied this concept but the Wcher brothers were the first to describe the combination of rotational gliding and rolling as being significant to understanding knee joint dynamics (Frankel \& Nordin, 1980). By marking the points of tibio-femoral contact throughout the range of knee motion the brothers made two important observations: (1) the point of contact on the tibial surface moved posteriorly as knee flexion increased, and (2) the distance between points of contact on the femoral condyle were twice as far as the corresponding distance between the points of contact on the tibial plateau. These results clearly demonstrated that a unique ratio of rolling to gliding existed within the knee joint; more specifically that the femoral condyle rolled and glided simultaneously over the tibial plateau as the knee moved throughout a range of motion.

More recently Müeller (1985) and Harding \& Blakemore (1980) have examined the kinematic principles of motion in the knee joint initially proposed by the Weber brothers. The results of their investigations indicated that an accurate plot of the contact poirts between the femur and tibia suggested that a quantifiable ratio between rolling and gliding existed. 
Although this ratio did not remain consistent throughout the entire ROM, a consistent 1:2 and 1:4 (rolling to gliding) ratio was evident at specific points through the range. Furthermore, these authors suggested that ACL dysfunction ultimately resulted in a breakdown of the normal kinematic synergy between rolling and gliding in the knee joint (Müeller, 1985).

\subsection{THE INSTANT CENTER PATHWAY}

The method used to describe the motion between the femur and tibia in the sagittal plane is called the instant center technique. This technique facilitates a description of the relative motion of two adjacent body segments and is obtained by the method of Reuleaux (1876) (Frankel et al., 1971). According to this method, the instant ce.is is found by identifying the displacement of two points on a link as the link moves from one position to another. Lines are drawn to connect the displaced pair of points on the link and the perpendicular bisectors of these lines are subsequently computed. The intersection of these bisectors locate the "centroid" or instantaneous center .

Gerber \& Matter (1983) suggested that abnormal shifts in the IC reflected in-vivo cartilage degeneration. Interestingly, specific increments of flexion and extension were found to have a pathological instant center of movement. Frankel et al., (1971) confirmed this observation. Operative exploration of internally deranged knees revealed that areas of degenerative cartilage in the femur and tibia contacted each other at exactly the range of movement for which the abnormal instant center had been found. The authors concluded that repetitive movement around an 
abnormal IC was the basis of "repetitive microtrauma" leading to degenerative arthritis of the knee.

Clinically, the pathway of the IC of a joint can be determined by taking successive roentgenograms of the knee in different positions from full extension to $90^{\circ}$ flexion by $10^{\circ}$ increments. The film is exposed and superimposed in pairs placing one tibia on top of the other. The Reuleaux (1876) method for locating the IC for each interval of motion is applied: (1) corresponding points on one of the films are located, (2) lines are drawn between these corresponding points, and (3) perpendicular bisectors of these lines are constructed to locate the centroid upon intersection. Frankel et al., (1971) applied this method to elucidate the mechanism by which tissue derangement caused mechanical derangement. In so doing thirty knees with known internal derangements were analyzed. Centroid abnormalities were observed in all cases. This seemed to indicate that the roentgenographic method employed by Frankel accurately characterized knee joint dysfunction and that any study investigating a similar phenomenon might well be served to include it in its protocol. Such is not the case.

Soudan \& Van Audekercke (1979) identified some limitations to Frankels' approach in studying human joint kinematics. First, it was unlikely that the $\mathrm{x}$-ray plate was positioned perfectly parallel to the joint when sequential radiographs were taken. In such instances a deformed view of the real planar motion would be registered on film. Second, inaccuracies arose when the link was not kept perfectly fixed during the procedure or when the joint moved out of the plane of motion from sequence to 
sequence. Finally, the application of the Reuleaux (1876) method to roentenography reduces the multi-planar joint to a uni-planar "hinge" joint. A more practical and accurate approach to the IC concept was suggested by the authors. It was felt that by computing the instant centers from data points and curve fitting methods inherent inaccuracies associated with the qualitative analysis of $\mathrm{x}$-ray interpretation could be avoided. A quantitative analysis of the IC concept is proposed in this study. Instant center of rotation curves are computed from three dimensional spatial coordinates and spline functions establish the best fitting curve for each rotation profile generated throughout a ROM.

\subsection{THE EFFECT OF ACL INSUFFICIENCY}

The ACL is an essential structure on which all mammals depend for knee joint stability. It is the keystone that controls the fluid complex flexion and rotational motion of the normal knee (Cabaud, 1983). Anatomically it is well established that the ACL consists of elastic and collagen fibres and is comprised of two principle parts: (1) a smaller anteromedial band (AMB) and, (2) a more bulky posterolateral band (PLB). Girgis et al., (1975) suggested the best way to visualize these two components was to think of them as being a narrow medial band with a bruad lateral part joined together (along their length) by soft material which permits them to move differently. This geometric configuration is responsible for the tightening and loosening of the different band portions throughout the ROM of the knee (King et al., 1986). Simply stated, each portion of the ACL contributes to different aspects of knee stability throughout a ROM and as such, is susceptible to different stresses. Amoczky (1983) confirmed this 
observation. He reported that when the knee was extended the PLB (B' B) of the ACL was taut while the AMB ( $\mathrm{A}^{\prime} \mathrm{A}$ ) remained moderately lax (Fig 1A). As the knee was flexed however, the femoral attachment of the ACL assumed a more horizontal orientation. This resulted in a tightening of the AMB ( $A^{\prime} A$ ) with a corresponding loosening of the PLB in comparison ( $B^{\prime}$ B) (Fig 1B). Other authors have investigated the geometry of the ACL and support the existence of anteromedial and posterolateral bands as being an accurate description of ACL anatomy (Furman et al., 1976; Hsieh \& Walker, 1976; Kennedy et al., 1974; Marshall \& Baugher, 1980; King et al., 1986). Such studies establish the unique relationship which exists between the spatial orientation of the ACL in flexion and extension and its function as a constraint of joint motion..

Herein lies the fundamental key to understanding the intricate kinematic synergy that exists between the femur and tibia; the realization that the rolling-gliding mechanism that characterizes femoral motion is directly affected by the shape and tension of the ACL band components as they change throughout a ROM. In flexion the AMB of the ACL is extremely taut. This facilitates gliding of the condyles on the tibial plateau by the anterior medial bands' ability to hold the femoral head in position as late flexion ensues (Girgis et al., 1975; Müeller, 1985). In extension however, the PLB becomes tightened relative to the AMB and femoral gliding is promoted. Again this may be attributed to the anchoring effect the posterolateral component of the ACL has on the femur in extension (Girgis et al., 1975; Müeller, 1985). Clearly this demonstrates that anatomic continuity of the two band components comprising the $\mathrm{ACL}$ directly influence the normal rolling-gliding synergy prevalent in the knee joint. 
SCHEMATIC DEPICTING TENSION

CHANGES IN THE COMPONENT BANDS

OF THE ANTERIOR CRUCIATE LIGAMENT

Extension

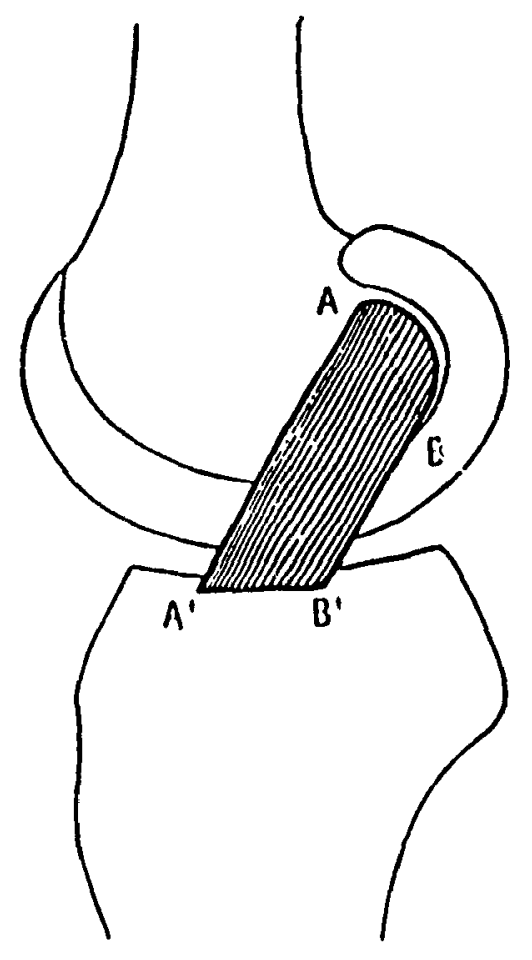

A
Flexion

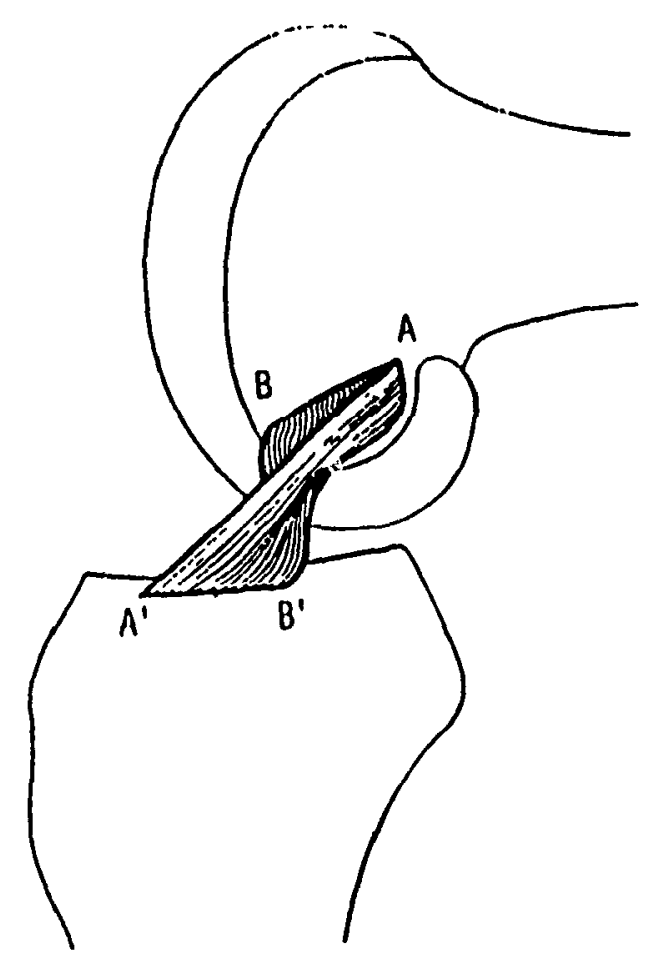

Figure 1 
Specifically, ACL dysfunction limits the gliding phenomenon and promotes rolling due to the inability of the ACL to effectively anchor the tibia on the femur.

Müeller (1985) described a four-bar linkage model that quantified the rolling-gliding ratio for an intact knee. A series of fourteen points were unevenly distributed on the suriace of the femoral condyle and tibial plateau respectively. It was determined that the distance covered by the condyle relative to the plateau was directly influenced by the geometrical configuration of the articulating bone and reflected a rolling-gliding mechanism. In extension the distance covered on the funur was nearly twice that on the tibia. In flexion the distance covered on the femur was approximately four times that on the tibia. Müeller suggested that in order for the condyles to accommodate the increased distances they must travel relative to the tibial plateau, supporting compliant tissue must serve as an effective anchor to prevent the the femur from literally rolling off the tibial plateau. A complex synergy between articulating bone and compliant supporting ligaments clearly existed to maintain the normal kinematic characteristics of the intact knee.

Müeller (1985) suggested the rolling-gliding ratio was $1: 2$ in extension and 1:4 in flexion and that the restraining properties of the ACL became compromised upon ligament dysfunction. Since excessive rolling is a unique characteristic of ACL deficiency, and since the kinetic synergy is compromised accordingly, it holds that migratory shifts of the IC of rotation can be quantified to reflect knee joint dysfunction in specific terms. Furthermore, if migratory shifts of the IC are "range specific" 
(occurring in late exterssion and flexion respectively), it holds that a three dimensional coordinate system that documents quasi-dynamic knee motion throughout a ROM might well identify pathological centroid motion within the confines of established X, Y, and Z axes. Gerber \& Matter (1983) and Frankel et al., (1971) reported that pathological movements of the IC resulted in joint compression and distraction at specific points throughout a ROM. The Genucom Knee Analyzer (GKA) quantifies femoral compression and distraction along a vertical ( $\mathrm{Y}$ ) axis . Therefore, disruption of the kinetic synergy between the femur and tibia for ACL deficient knees will primarily be reflected by translations of the IC along the $\mathrm{Y}$ axis defined in this study. Instant center coordinates were also quantified in this study between $100^{\circ}$ and $70^{\circ}$ along a sagittal $(Z)$ axis. Thes: "range specific" parameters were selected for two reasons: (1) preliminary data did not seem to characterize the deficiency in quantifiable terms throughout the entire ROM, and (2) existing literature stated that the ACL was functionally most taut at the ends of its range of motion (Girgis et al., 1975; Arnoczky, 1983).

\subsection{PASSIVE STRUCTURES}

The physiological and mechanical properties of various knee ligament structures have been described in detail by many authors. While there is general agreement that the primary function knee ligaments is to limit the motion between the tibia and femur, conflicting results abound in the literature concerning the definitive influence the ACL has on joint stability. Fukubayashi et al., (1982) explored the anterior-posterior motion of the tibia as it moved from $0^{\circ}$ to $90^{\circ}$ flexion for nine normal cadaver knees at 
$15^{\circ}$ increments. Tibial displacement, rotation, and torque variables were quantified in response to applied loads of up to 125 Newtons. Measurements reported of intact knees consistently showed that an anterior force produced an internal tibial torque and subsequent internal tibial rotation. Selective sectioning of the ACL resulted in over doubling the amount of anterior displacement when compared to intact knees.

Marquette (1988) described the debilitating anterolateral rotatory instability injury. He suggested such injuries were brought about by two events: (1) increased anterior drawer of the tibia, and (2) a shifting of the knee axis to a point further medial. This medial shifting of the knee center created an increased lever arm relative to the lateral tibial condyle and resulted in the production of a larger angular radius. The combination of an increased lever arm with a corresponding increase in anterior tibial displacement resulted in anterior subluxation of the lateral tibial plataeu. Ahmed et al., (1987) determined the contribution of the ligaments to the load-resistance mechanism of the knee between $40^{\circ}$ and $90^{\circ}$ flexion. The tension generated in selected fiber bands of the four major knee ligaments were measured in-vitro. Ligament strain analysis revealed the $A M B$ and PLB of the ACL lengthened in internal rotation. Such investigations demonstrate that in addition to acting as an anterior-posterior stabilizer, an important function of the $\mathrm{ACL}$ is to act as a restraint to internal tibial rotation.

Markolf et al., (1978) suggested a necessary prerequisite for evaluating knee integrity involved the analysis of force-displacement curves that traced the knees' structural response to applied loads during a clinical 
examination. Such tracings would permit the calculation of knee stiffness; a parameter that in the past had been "felt" by clinicians, but never actually quantified. He supported the inclusion of stiffness measures as a necessary parameter to define knce stability in specific terms.

Shoemaker \& Markolf (1985) measured the effects of serial sectioning of the medial collateral ligament (MCL), the $\mathrm{ACL}$, and finally the effects of combined sectioning of both ligaments on anterior-posterior forcedisplacement response curves for seven cadaver knees. Effects of joint load on ligament deficient knees were evaluated on the bases of stiffness and laxity parameters. Stiffness was defined as the slope on the forcedisplacement curve while laxity was defined as the distance in millimetres the tibia was displaced from the femur in the sagittal plane. Initial sectioning of the MCL had no significant effect on total absolute laxity; however, when the ACL was sectioned first there were large increases in anterior laxity and corresponding reductions in anterior stiffness.

In the same study changes in the stiffness patterns of the curves obtained were also noted. The authors observed a definite "break-point" in the curve which was marked by a sudden decrease in stiffness. This phenomenon occurred midrange and initiated the non-linear response curves characteristic of knee dysfunction. They suggested that the congruent surface geometry and contact forces that normally restrict anteriorposterior displacement were overcome by loads applied mid-range (Shoemaker \& Markolf, 1985). This resulted in an observable "breakpoint" in the response curve and was attributed to the support of the primary restraining ligament. It appears therefore, that quantification of 
this midrange stiffness parameter is necessary to provide additional information regarding ligament integrity.

Butler et al., (1980) specifically identified the primary and secondary stabilizers contributing to knee stability. By measuring the restraining force which developed in individual ligaments during tibial displacement the primary "workers" and secondary "helpers" in resisting joint displacement were characterized. In this way the relative importance and function of a single ligament was assessed in terms of the per cent of total restraining force which it provided. The authors concluded from their studics on restraining forces that for each of the planes of knee motion, only one or two ligaments acted as primary restraint. The other ligaments provided only a secondary restraint. Specifically it was discovered that the ACL provided $85 \pm 1.9 \%$ of the total anterior restraining force at $90^{\circ}$ flexion and $87 \pm 1.8 \%$ restraining force at $30^{\circ}$ flexion. Furthermore, it was determined that we posterior cruciate ligament (PCL) provided virtually no restraint to anterior translation of the tibia at all flexion angles.

The results of these investigations support the inclusion of stiffness and laxity parameters as being appropriate criterion variables for characterizing knee joint dysfunction. This sterns from the established importance the $\mathrm{ACL}$ has in promoting knee integrity and the need to quantify ACL dysfunction in specific terms. 


\subsection{EVALUATION OF KNEE LAXITY}

The Lachmans test has become recognized as being the most reliable and non-invasive clinical tool for determining the integrity of the ACL (Gurtler et al., 1987). Gurtler et al., (1987) reviewed the clinical and operative findings of 250 knees presented for surgery with internal derangements. The operative findings were correlated with the classical anterior drawer sign at $90^{\circ}$ and $30^{\circ}$ flexion. All knees having isolated operative findings of $\mathrm{ACL}$ tears resulted in a positive Lachmans test performed at $30^{\circ}$ flexion. The authors reported that the Lachmans test was the only clinical test to consistently elicit joint symptoms associated with ACL dysfunction.

Rossenberg \& Rasmussen (1984) further clarified the functional characteristics of the ACL during clinical test in-vivo. Normal subjects were examined using arthroscopy and a special probe which was designed to measure tension in the material it contacted. The tip of the probe was calibrated in millimetre increments and a spring scale mechanism on the handle allowed the surgeon to apply a known force to the ligament. The amount of tibial displacement was simultaneously recorded. Tension in the ACL was evaluated before and during testing with a drawer test performed at $90^{\circ}$ and $15^{\circ}$ flexion respectively. Baseline tension was reported as being consistently greater at $15^{\circ}$ flexion. Results of this study support the specificity of the Lachmans test as an indicator for assessing ACL integrity.

Gurtler et al., (1987) proposed three reasons for the superior results obtained in evaluating $A C L$ dysfunction with the Lachmans test. First, the 
test is performed in a position of comfort for the subject with the knee slightly flexed between $10^{\circ}$ and $30^{\circ}$ degrees. Second, protective spasm of the hamstring muscles is negated with the joint extended. Such spasms often inhibit anterior translation of the tibia on the femur. Finally, the geometric continuity of the joint in extension is such that the anterior aspects of the femoral condyles are in a position which does not obstruct anterior translation.

\subsubsection{THE NEUTRAL POINT}

Despite widespread agreement that knee evaluation is most accurately performed with the knee positioned between $15^{\circ}$ and $30^{\circ}$ flexion, there is little agreement regarding the clinical significance for establishing a "neutral zone" prior to evaluation. Furthermore, researchers are hard pressed to define this parameter in specific quantitative or qualitative terms. Butler et al., (1980) defined "neutrality" as being the point along force-displacement curves where stiffness parameters were "smallest". Fukubayashi et al., (1982) declared the "inflection point" (a transitory plateau between anterior-posterior load-displacement curves) best represented the neutral zone.

Torzilli et al., (1981) examined a "stable" population of 18 males and 11 females in an attempt to establish normal criteria that would describe knee motion. In their study the neutral point was defined in general terms as being the "unloaded" position. They suggested the establishment of the neutral point was a critical factor for the accurate diagnoses of knee deficiencies in order to prevent the "false positive" drawer phenomenon 
from being elicited. This situation can result in the mis-diagnoses of an $\mathrm{ACL}$, when in reality, it is the PCL that is structurally deficient. Confusion stems from "tibial sags" which occur in response to the extreme posterior position assumed by the shank (due to gravitational forces) at the on-sat of the clinical evaluation. When anterior loads cause the shank to "drawer forward" in such cases, the examiner may well misinterpret the positive drawer as being symptomatic of ACL dysfunction. Torzilli et al., (1981!) demonstrated that such a phenomenon was unlikely to occur for subjects presented with intact knees. Using a series of roentgenograms the authors determined that mean differences in neutral positions between "stale" male and female subjects were not statistically differnt. Furthermore, the difference in neutral zones bilateraly were found to be equally distributed in the anterior and posterior direction. Finally, the authors reported negligible differences to exist between right and left knees for the motion parameters obtained for all subjects. This finding supports the assumption of bilateral symmetry made for the subjects participating in this study.

Despite the confusion in defining the neutral point in specific terms, the need for establishing a neutral zone as a prerequisite for accurately diagnosing the acutely injured knee is apparent. However, for patients exhibiting chronic instability associated with third degree isolated ruptures; of the ACL, the need for establishing a definitive neutral point assumes less significance due to the inability of the tibia to "sag posteriorly" (Butler et al., 1980). 


\subsection{ACTIVE STABILIZERS}

The surrounding musculature and tendons constitute active stabilizers and function to promote knee joint stability (Baretta et al., 1986). Soiomonow et al., (1987) studied the ligament-muscle reflex arc. He exposed cat ACL's by wrapping them with a wire which allowed the investigators to "stress" the ligament. EMG electrodes were inserted in the quadriceps and hamstrings to monitor activity. During the $\mathrm{ACL}$ loading cycle there were no EMG discharges from the supporting musculature. When the applied loads were substantially increased to forces approaching structural rupture, the hamstrings "discharged" vigorously while the quadriceps remained inhibited. This "pain induced" type of reflex seemed to protect the ligament by recruiting additional posterior pull from the hamstring to sustain the applied load. However, this reflex arc mechanism could not provide sufficient protection to the knee when the applied load exceeded the maximum sustainable limits of the ligaments and muscle.

Solomonow et al., (1987) described another type of "pseudo reflex" arc which originated from the joint muscle receptors. He termed this arc "joint capsule-hamstring reflex". As ACL deficient subjects performed maximal isokinetic extensions at $15 \% \mathrm{sec}$, the knee was observed to subluxate anteriorly at $40^{\circ}$ of flexion. The resulting EMG activity revealed quadriceps inhibition and hamstring stimulation. This suggested that this particular reflex may not actually prevent subluxation, but more accurately correct for it. The author proposed such corrective mechanisms were learned and as such, could not be considered as being truly reflexive. Furthermore, the authors noted that because subjects had ruptured their 
$\Lambda C L ' s$, such reflex excitation could not have come from receptors within the ACL, but rather from receptors in the joint capsule or hamstrings themselves.

Other investigations have studied the effects of musculature on stiffness and laxity parameters. Of the numerous studies performed, there is general agreement among researchers that the surrounding musculature increases joint stiffness and decreases joint laxity. Johnson and Hull (1988) found that resisting musculature could increase internal rotatory stiffness by $100 \%$ and external rotatory stiffness by $320 \%$. This marked increase in stiffness was accompanied by a $20 \%$ reduction in rotatory laxity.

Louie \& Mote (1987) performed a detailed study on the partial and compound effect of the various musculature that cross the knee joint. The contributions of maximally contracting quadriceps was found to increase joint stiffness by $218 \%$ over the passive muscular condition at $90^{\circ}$ flexion. An increase of $314 \%$ in stiffness was recorded at $10^{\circ}$ flexion when the quadriceps were active. These results seemed to suggest that the contribution of musculature to knee stability is sensitive to flexion angle. More specifically, these data suggests that a flexed knee is more vulnerable 10 sustaining an injury than the extended position since joint stiffness is substantially reduced in flexion. The contribution of the hamstrings to the maintenance of joint stability was also examined by the same authors. At $90^{\circ}$ flexion, contraction of the hamstrings was found to reduce total laxity to $24 \%$ of passive laxity conditions while the quadricep activity reduced passive laxity conditions to $77 \%$. This suggested that the hamstring contribution to knee stability was three times greater relative to the 
contribution of the quadriceps at $90^{\circ}$ flexion. A similar trend was observed in extension.

These studies are significant because they establish the relationship between knee joint stability and the contribution of supporting musculature to stiffness and laxity parameters. Specifically, it was determined that knee joint stiffness increased while joint laxity decreased when the hamstrings and quadriceps were activated. The implication of this finding to the assumption of muscular passivity is an important one. It is evident that inadvertent contraction of surrounding musculature by subjects tested in this study will manifest in the motion parameters quantified by the GKA. Specifically, muscular activity will be reflected by corresponding decreases in laxity and corresponding increases in stiffness parameters for subjects tested bilateraly. It is essential therefore, that stiffness and laxity parameters be included as descriptors of joint motion for two reasons: (1) to further characterize the nature and extent of the injury. and (2) to assess the extent of muscular activity generated by subjects throughout the testing protocol.

\subsection{INSTRUMENTED DEVICES}

The need for an objective knee testing device is apparent. Presently, two testing devices commonly used by clinicians to quantify knee laxity include the Stryker Knee Laxity Tester and the KT 1000 Knee Arthrometer. Both devices measure anterior-posterior tibial excursions at a variety of flexion angles however, the data generated by these instrumented devices are suspect for three reasons. Foremost, tibial translations are reduced to uni- 
planar measures and do not reflect the three dimensional characteristics of knec joint motion. Secondly, soft tissue is not compensated for anywhere in the testing procedure. As a result, measurement errors due to the presence of soft tissue between the measurement apparatus and the bones and ligaments can not be accounted for. Finally, both devices quantify laxity as a function of tibial translation relative to the amount of patellar excursion. Clearly the validity of a measure which does not account for the subject specific tracking patterns of the patella is suspect.

\subsubsection{THE GENUCOM KNEE ANAL YZER}

The Genucom Knee Analyzer (GKA) is a computer based system designed to produce in-vivo force-displacement data. It consists of a force plate and a three dimensional goniometer linkage system interfaced with a microcomputer. Unlike the uni-planar measures of tibial displacement obtained by the Stryker and KT 1000, the GKA accommodates the threedimensional structure of the knee joint by quantifying orthogonol multiplanar tibial motion. The six degrees of freedom linkage electrogoniometer measures displacement responses to forces applied in six directions. Translational motions are quantified by anterior-posterior, medial-lateral, and proximal-distal applied loads. Rotational motions are quantified by loads eliciting varus-valgus, internal-external, and flexion-extension movements. Additionally, an inherant soft tissue compensation procedure accounts for femoral motion associated with tibial manipulation. In this way the relative position of bones to examiner applied loads can be quantified by accommodating bone displacement typically associated with soft tissue compliance in a clinical examination. The need for such 
compensation protocols is apparent. Torizilli et al., (1981) suggested that objective measures must somehow compensate for femoral motion associated with soft tissue compliance.

\subsection{SOFT TISSUE COMPENSATION PROCEDURE}

Multi-planar tibial motion is described in relationship to a three dimensional coordinate system defined by the GKA. An orthogonol matrix establishes an $\mathrm{X}, \mathrm{Y}$, and $\mathrm{Z}$ plane in which all motion parameters are defined and characterized. By convention the $X$ plane defines an orthogonol $\mathrm{Y}$ axis and is reflected by femoral compression and distraction. The $\mathrm{Z}$ plane defines an orthogonol $\mathrm{X}$ axis and is reflected by medial-lateral femoral motion. The $\mathrm{Y}$ plane defines an orthogonol $\mathrm{Z}$ axis and is reflected by anterior-posterior motion. These axes interface to quantify joint movement in all planes simulianeously and are defined relative to a fixed femur (Fig 2). With the electrogoniometer measuring femoral motion, the certified Genucom examiner applies translational and rotational loads to the femoral condyles. The GKA records the amount of femoral displacement associated with the examiner applied loads to a fixed femur. These loads are applied on two separate occasions and the resulting forcedisplacement curves aresaved by the device and compared to determine the reliability of the obtained measures.In so doing, the stiffness characteristics of the displaced femur within the soft tissue confines of the thigh are also computed and saved. (It is essential that comparisons be made between stiffness characteristics and force-displacement curves generated for each subject and not with a pre-established curve reflecting "ideal tissue density" as adipose : muscle ratios are subject specific with high inter-subject 


\section{THREE DIMENSIONAL COORDINATE}

SYSTEM DEFINING DIRECTIONAL

AXES ON THE GENUCOM KNEE ANALYZER

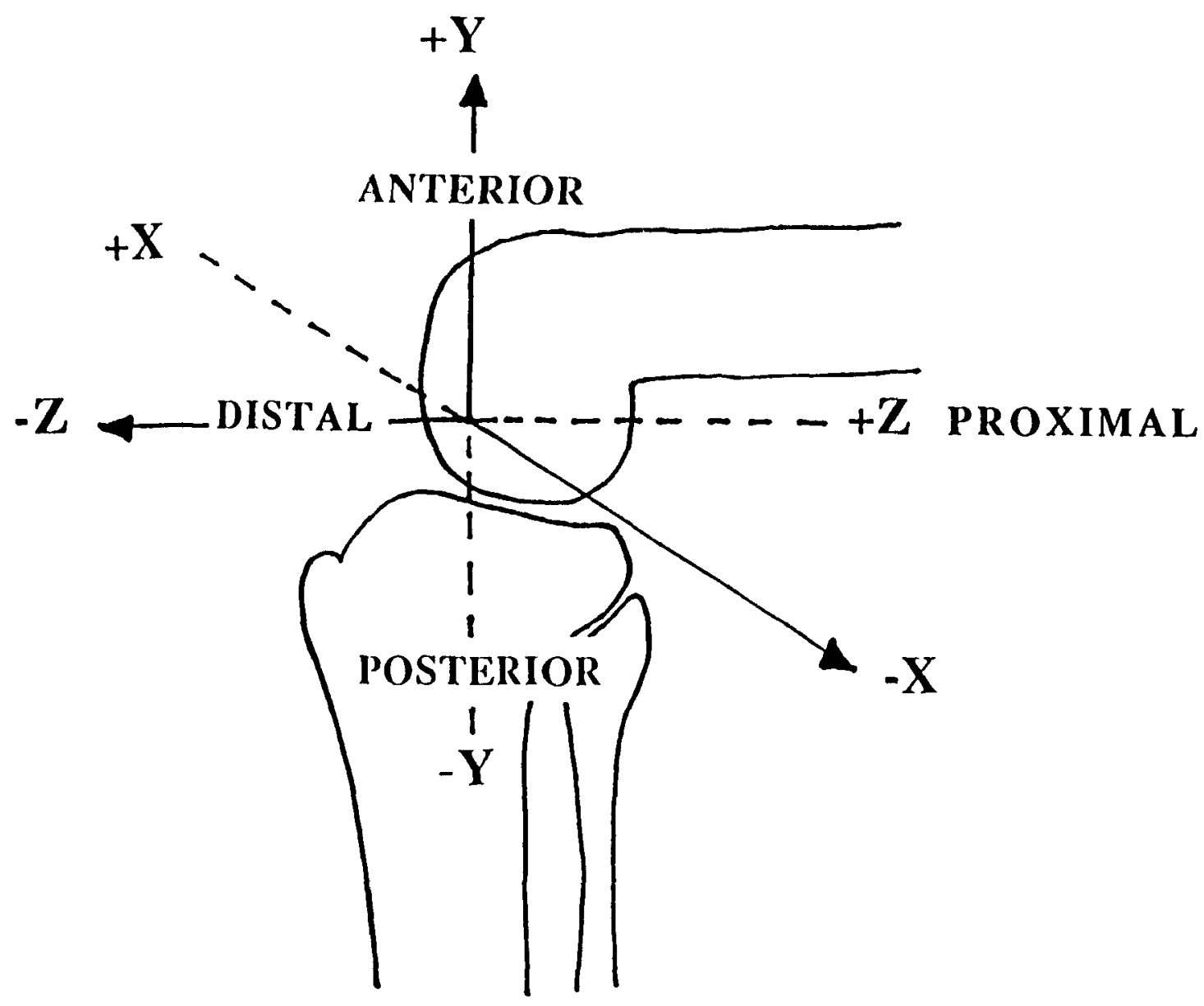

Figure 2 
variability). A Pearson Product Moment Coefficient correlates the number of discrepant stiffness measures obtained at identical force values gencrated between force-displacement curves. The number of measures that differ by less than $2 \mathrm{~mm}$ per unit force are reported to the examiner as a total percentage of correlated values. High percentages therefore reflect similar compression and stiffness characteristics between compensation trials and support the reliability of the obtained measures. This information is stored on the GKA and reflects the magnilude of femoral displacement associated with translational and rotational loads applied at the femoral condyles. Any are subject specific with high inter-subject variability). A Pearson Product Moment Coefficient correlates the number of discrepant stiffness measures obtained at identical force values generated between force-displacement curves. The number of measures that differ by less than $2 \mathrm{~mm}$ per unit force are reported to the examiner as a total percentage of correlated values. High percentages therefore reflect similar compression and stiflness characteristics between compensation trials and support the reliability of the obtained measures. This information is stored on the GKA and reflects the magnitude of femoral displacement associated with translational and rotational loads applied at the femoral condyles. Any subsequent examiner applied load to the tibia will inevitably result in femoral motion accompanying relative tibio-femoral motion. This results in a "gross" measure of relative bone displacement that is clearly affected by the amount of tissue compliance within the thigh. The effect of this gross measure is accommodated by statistically subtracting the stiffness. displacement measures associated with femoral compression at the on-set of the compensation procedure to yield a "net" tibial displacement measure. 
The GKA has been demonstrated as being effective in identifying joint deficiencies (Oliver \& Coughlin, 1987; Brien \& Hoshizaki, 1986). Hoshizaki \& Matthews (1989) established the day to day variability of measures obtained with the device. Inter-tester variability was also examined. Results of their investigation demonstrated that motion parameters obtained on a battery of clinical tests remained consistently stable between trials and between days. Furthermore, inter-tester variability was demonstrated to be statistically insignificant on six of the eight dependant measures. In the two cases where tester variability did exist, the values obtained were well within the functional range of knee laxity (Hoshizaki \& Matthews, 1989). All measures were evaluated at the 0.05 confidence level. The authors concluded that the GKA provided reliable data between trials, between testers, and from day to day.

\subsection{SUMMARY}

The destabilizing effects of ACL deficiency on the functional and biomechanical characteristics of knee motion were described. However, controversial information abounds in the literature regarding the role this important structure assumes in promoting joint integrity for three reasons: (1) in-vitro methodologies negate the true physiological and anatomical continuity of the knee joint by their invasive design, (2) the three dimensional nature of the joint is difficult to model and has resulted in the assumption that knee motion may be reduced to uni-planar measures, and (3) interpretations of proprioceptive techniques attempting to describe joint dysfunction are subjective. In light of these limitations it becomes clear that quantification of motion parameters attempting to document the 
destabilizing effects of ACL dysfunction must meet two essential criteria: (1) measures must reflect the corresponding structural deficits associated with the injury, and (2) measures must attempt to characterize the dynamic interaction which is known to exist between bone and compliant ligaments. 
CHAPTER III

METHODS AND PROCEDURES

\subsection{INTRODUCTION}

Clinical techniques for evaluating knee stability are subjective and often result in discrepant diagnoses (Torzilli et al., 1981). In an attempt to identify a more effective in-vivo approach of characterizing knee joint dysfunction, it is imperative to adopt an objective mechanical measure of knee motion (Markolf et al., 1978). Ideally objective measures should attempt to replicate and assign a value to the parameters "felt" clinically. Additionally, such measures should reflect the dynamic interaction which exists between articulating bone and compliant ligaments (Huiskes et al., 1987).

The purpose of this investigation therefore, was to develop an objective, non-invasive methodology that would document and characterize the effects of isolated ACL dysfunction on knee motion. Two essential components of the study involved: (1) the quantification of a variety of three dimensional motion parameters to confirm the extent of a diagnosed deficiency, and (2) the documentation of the "quasi-dynamic" link synergy reflecting relative tibio-femoral motion throughout a ROM in the sagittal plane. In this way synergistic motion parameters were identified for instant center of rotation coordinates obtained for intact and involved knees. 


\subsection{SUBJECT SELECTION}

A total of twenty subjects were selected to participate in this study. Male and female adults between the ages of 18 and 33 years were examined by an experienced Orthopaedic Surgeon. Clinical tests involving proprioceptive joint manipulation identified those subjects having uni-lateral, second degree ruptures of the ACL according to a modified scale proposed by Houghston et al., (1976) (Appendix A). In this classification of joint instability a grade two injury was characterized by an anterior tibial laxity range of 5 to 9 millimetres. A grade three injury was characterized by an anterior tibial laxity range of 10 to 15 millimetres. Confirmation of the subjects clinical instability was accomplished by an arthroscopic examination performed by the same surgeon in thirteen of the twenty cases. All subjects were then evaluated on the GKA by a certified examiner. A series of three bilateral anterior drawer tests were then performed on each subject. Anterior terminal laxity parameters were quantified at $130 \pm 5$ Newtons. Subjects were then screened for protocol completion based on the following criteria; (1) clinical examination of the contralateral knee revealed an absence of previous ligament disruption. (This was confirmed by the subjects' disclosure of previous non-treatment or injury to the joint), and (2) the presence of bilateral laxity asymmetries between involved and intact knees of 5 millimetres or greater. Markolf \& Amstutz (1987) reported that ACL dysfunction was characterized by mean contralateral asymmetries of five millimetres. This inclusion criteria reduced the sample size to fifteen subjects. 


\subsection{DESCRIPTION OF THE STUDY}

The experiment consisted of three parts. The first part of the study established the reliability of the GKA to accurately quantify the instant center (IC) of rotation motion parameter throughout a ROM. The second part of the study objectively established the presence of a subjectively diagnosed deficiency. In so doing, those subjects not exhibiting anterior bilateral laxity asymmetries of five millimetres or more were excluded from continuing on with the third part of the investigation; a kinematic analysis of relative tibio-femoral motion. In this part of the investigation the method of Reuleaux (1876) was applied to compute IC of rotation displacement parameters for intact and involved knees throughout a ROM.

\subsubsection{PART I}

The shape reliability of the IC joint rotation patterns were determined initially for two reasons: (1) the presence of a consistent shifting phenomenon of the IC throughout a ROM would establish the stability of the three dimensional spatial coordinates used to compute the parameter according to the method of Reuleaux (1876), and (2) the curves facilitated the identification of dependant measures at the ends of the range that reflected the dynamic synergy between the femur and tibia. The reliability of the GKA to accurately quantify inter-trial and within-subject variability of the IC of rotation parameter was then statistically assessed. The following methodology elucidated the measures of interest. 
One subject was evaluated. No previously diagnosed structural knee joint deficiency was documented. The GKA was used to obtain three dimensional spatial coordinates of three anatomical bony landmarks: (1) the tibial tuberosity, (2) a point on the tibia! crest 15 centimetres inferior to it, and (3) the medial epicondyle of the femur. The proximal aspect of the shank was loaded with an anteriorly applied force of 120 Newtons as the tibia moved throughout a ROM. The femur remained fixed as the shank was positioned from $100^{\circ}$ flexion to $-10^{\circ}$ extension by $10^{\circ}$ increments. Quantification of anatomical spatial coordinates every $10^{\circ}$ provided the preliminary data from which to compute the IC of rotation coordinates. Ten trials were performed consecutively on two sequential days. Reuleaux coordinates generated in the sagittal plane were plotted against flexion angle and a spline function established the best fitting curve. In this manner a total of twenty unique graphs were plotted to depict the migration shifts of the IC of rotation parameters throughout a ROM. Pearson Product Moment Correlations (PPMC) determined the shape-reliability of the graphed profiles within trials and between days. High correlations indicated good shape-reliability. The variability of relative IC coordinates were evaluated at two distinct ranges along the curve: (1) in flexion between $100^{\circ}$ and $70^{\circ}$, and (2) in extension between $30^{\circ}$ and $0^{\circ}$ degrees. IC displacement coordinates were identified at the ends of the range for two reasons: (1) secondary constraining and supporting structures interface midrange to maintain joint stability (Girgis et al., 1975), and (2) ligament stiffness is compromised greatest at the ends of the range (Amoczky, 1983). Since IC parameters are influenced by the relationship between the relative 
position of link segments and ligament stiffness, it holds that pathological migration patterns of IC coordinates would manifest predominantly in those situations in which the structural integrity of the ACL was compromised greatest; at the ends of the range. For this reason IC of rotation displacement parameters were quantified in specific terms along the $\mathrm{Y}$ axis in extension and along the $\mathrm{Z}$ axis in flexion. Specifically, the difference between the minimum and maximum Reuleaux coordinates obtained between $30^{\circ}$ and $0^{\circ}$ degrees were statisticaly assessed for reliability along the $\mathrm{Y}$ axis and reflected link synergy in extension. Similarly, the mean Reuleaux coordinate obtained along the $\mathrm{Z}$ axis (between $70^{\circ}$ and $100^{\circ}$ degrees of flexion) subtracted from the maximum Reuleaux coordinate (obtained between $20^{\circ}$ and $50^{\circ}$ ) reflected link synergy in flexion. Means and standard deviations of the calculated IC parameters established the presence of a quantifiable and consistent migration phenomenon at the ends of the range. A two-tailed t-test evaluated significance at the 0.05 confidence level.

\subsubsection{PART $\amalg$}

It was imporant to confirm the nature of a subjectively diagnosed deficiency and to establish the extent of clinical laxity asymmetry between knees for each of the twenty subjects tested for two reasons: (1) it provided objective documentation from which to assess the structural specificity of the injury, and (2) it established a quantative rationale for including only those subjects adhering to the joint symptom criteria of bilateral laxity asymmetry. These subjects were identified by 
performing a series of bilateral clinical tests on each of them using the GKA. A certified examiner conducted the tests. Stiffness and laxity motion parameters were quantified Those subjects exhibiting bilateral anterior laxity asymmetries of five millimetres or more were accepted for protocol completion.

\subsection{THE GENUCOM TEST}

The Genucom system is controlled by a software package developed by its manufacturer (Farro Inc.). A six component force dynamometer is built into the seat of the chair and measures the forces, moments, and torques applied by the examiner to the knee joint. A six degrees of freedom linkage goniometer attaches distally to the tibia in order to measure the joint angle and relative tibial-femoral displacements associated with the application of these loads. These measures are accurate to plus or minus one millimetre on translation, plus or minus one degree on rotation, and plus or minus one pound on any applied force (Oliver \& Coughlin, 1987). Testing began by "installing" the subject into the device. This involved seating the subject into the Genucom chair in a reclined, supine position. The trunk, hips, and femur were subsequently stabilized. This was accomplished by restraining the subjects' thigh with three "restraining pads". The lateral thigh pad was secured first. A 130 Newton force was then applied to the medial thigh pad and tightened. A 90 Newton posteriorly directed force was then applied to the superior thigh restraint pad and tightened. More subtle femoral motions were accounted for by the ensuing soft tissue compensation protocol which followed the subject installation 
procedure. This ensured that motion parameters reflected "relative" bone displacement and not "gross" tibial displacement; the latter measure being influenced by femoral tissue compliance within the thigh upon joint manipulation. Soft tissue measures eliciting correlation percentages of $90 \%$ or higher between tissue compensation trials were prerequisites for continuing the testing protocol.

The effective knee center was then computed in the following manner. Seven anatomical landmarks were identified on the shank and femur respectively. These included: two points on the tibial crest, the tibial tuberosity, the medial and lateral tibial plateaus, and finally the medial and lateral femoral condyles at half-width (Fig 3). Each landmark was subsequently assigned an $\mathrm{X}, \mathrm{Y}$, and $\mathrm{Z}$ coordinate relative to a computer established origin. A digitization procedure identified the spatial location of each landmark in three orthogonol planes. The effective knee center was then computed based on the relationship between anatomical landmarks in the transverse, frontal, and sagittal planes. The intersection of these spatial coordinates established the effective knee center of the joint in a neutral (unloaded) position. The location of the medial and lateral tibial plateaus were also quantified. They were determined as being positioned one centimetre from the knee center computed in the transverse plane. In this way subluxation of the lateral tibial plateau (ATLP) could be expressed as anterior translation of the plateau center in response to an applied load. Position coordinates of the effective knee center were also expressed in response to examiner applied loads relative to the knee center initially computed. This information was stored on a patient disk which inserted into one of two 


\section{ANATOMICAL LANDMARKS DIGITIZED BY THE GENUCOM TO COMPUTE THE EFFEC'TIVE KNEE CENTER}

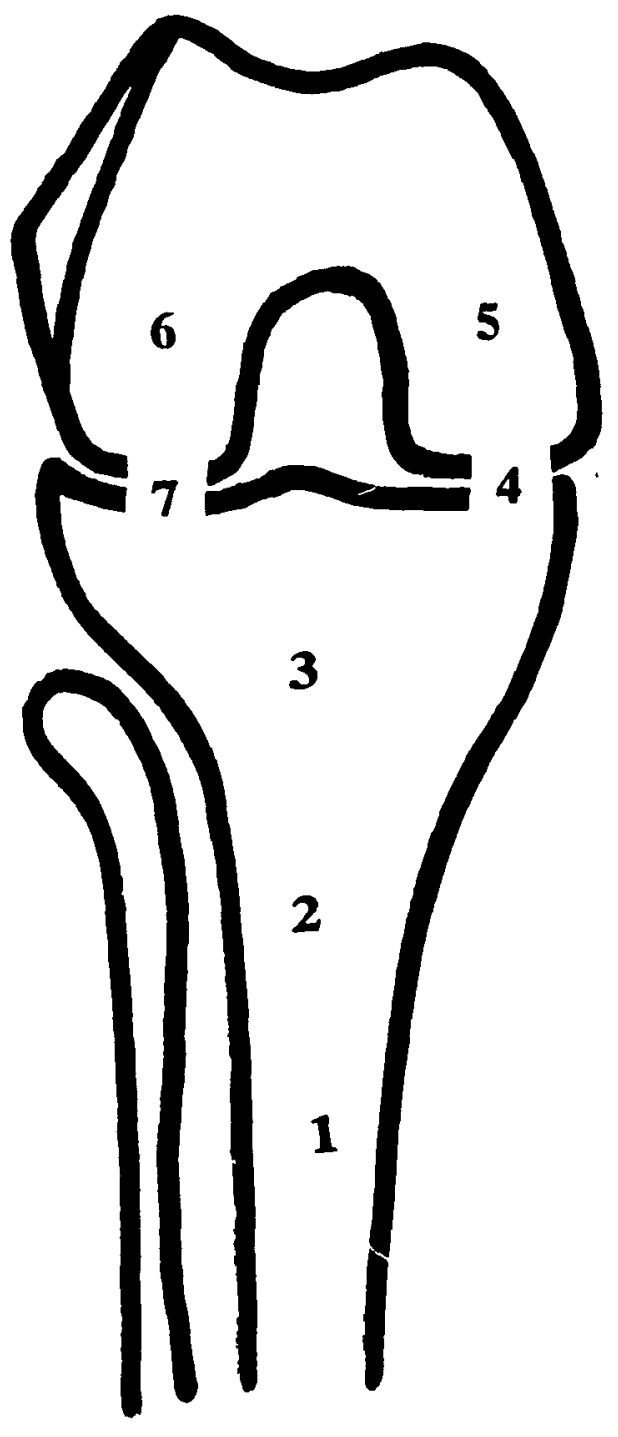

1 \& 2: Tibial Crest

3: Tibial Tuberosity

4 \& 7: Tibial Plateau

5 \& 6: Femoral Condyles at Half-Width

Figure 3 
disc drives at the base of the machine. The force applied to the shank was monitored and simultaneously recorded with tibial displacement measures associated with the translation. This information was saved on a patient diskette and graphed to depict the force-displacement and torque-rotation response curves elucidated for each subject after the soft tissue displacement measures were statistically removed from the data.

\subsubsection{TESTING PROCEDURE}

Once installed into the GKA and the soft tissue compensation procedure completed, each subject experienced a series of bilateral clinical tests. Three anterior drawer tests were performed by a certified examiner with the knee flexed: (1) at $90^{\circ},(2)$ at $90^{\circ}$ with the foot externally rotated $10^{\circ},(3)$ at $30^{\circ}$, and (4) at $20^{\circ}$ degrees.An anterior load of $130 \pm 5$ Newtons was then applied to the proximal tibia in each instance. The GKA recorded the relative displacements associated with the incremental increases in force at a frequency of $15 \mathrm{Hertz}$. Anterior terminal laxity $(\mathrm{AL})$ parameters were quantified at $130 \pm 5$ Newtons and reflected total anterior translation of the tibia relative to a fixed femur at each flexion angle. Edixhoven et al., (1987) reported that second and subsequent cycles of drawer curves were always reproducible while first-cycle reproducability was questionable for force-displacement curves obtained with instrumented devices. For this reason three trials were performed for each drawer test to "precondition" subjects to the loads they would experience. The terminal force value of $130 \pm 5$ Newtons was chosen for two reasons: (1) it approximated values applied by clinicians evaluating knee joint 
stability by proprioceptive methodologies, and (2) it reduced the potential of further injury to the ACL associated with the application of higher loads.

Ante:ior midrange stiffness (AMRS) was quantified from forcedisplacement data obtained by anterior drawer tests for two reasons: (1) the parameter was thought to reflect the support provided by the primary restraining ligament and (2) stiffness parameters obtained midrange most accurately reflect the tautness of the ACL (Shoemaker \& Markolf, 1985).This measure was evaluated between 40 and 60 Newtons. The stiffness value was computed as the inverse slope connecting the two data points closest to the 40 and 60 Newton force values obtained in the testing procedure. In other words, stiffness was represented as the inverse of a line established by two data points: one at 40 Newtons and one at 60 Newtons. If for any reason these specific values could not be identified on the load-displacement curves generated, the two data points closest in proximity to them were used in the analysis (provided they fell between the 40 and 60 Newton range).

A series of three internal-external tibial rotation tests were then performed with the subjects' knee positioned at $90^{\circ}$ flexion. A torque of $11 \mathrm{~N}-\mathrm{m}$ was then applied in each instance unless the subject experienced pain in response to the applied load. In three such cases the wrque loads were reduced to accommodate the individual pain thresholds of the subject. Internal tibial rotations (ITR) were measured in degrees in response to incremental increases in torque values generated internally and externa!ly. Additionally, anterior translation of the lateral tibial 
plateau (ATLP) was simultaneously recorded in response to the $11 \mathrm{~N}$-m torque load.

The laxity and stiffness parameters obtained in this part of the investigation were used to supplement a thorough patient examination and history. As such, the quantification of these motion parameters served primarily a descriptive function as opposed to the selective function of anterior laxity parameters obtained earlier in the protocol.

\subsection{EXPERIMENTAL DESIGN}

One-way ANOVA's established statistical significance between knee condition (Factor A) on each of the dependant measures: AL, AMRS, ATLP, and ITR. Two levels of knee condition were assessed: involved and intact. The dependant variables were statistically evaluated at the 0.05 confidence level.

\begin{tabular}{|l|c|}
\hline \multicolumn{2}{|c|}{ FACTOR A: KNEE CONDITION } \\
\hline Involved & Intact \\
\hline
\end{tabular}

Three two factor ANOVA's assessed the laxity and stiffness characteristics of the subject profile across flexion angle. Factor A included four levels of flexion angle while Factor B included two levels of knee condition. In this manner AL, AMRS, and ATLP were 
evaluated for significance at the 0.05 confidence level. A Tukey PostHoc means comparison identified the locus of the interaction between Main Effects for each dependant measure.

\begin{tabular}{|c|c|c|c|c|}
\hline FACTOR B & \multicolumn{4}{|c|}{ FACTOR A: FLEXION ANGLE } \\
\hline KNEE CONDITION & $20^{\circ}$ & $30^{\circ}$ & $90^{\circ}$ & $90^{\circ} \& 10^{\circ} \mathrm{ER}$ \\
\hline Intact & & & & \\
\hline Involved & & & & \\
\hline
\end{tabular}

\subsection{PART U}

The third part of the investigation documented the IC joint rotation patterns of intact and involved knee joints according to the method of Reuleaux. Three dimensional spatial coordinates obtained with the GKA were used to compute this motion parameter according to the following methodology. Each subject was installed into the Genucom. The femur was secured and three anatomical landmarks were identified on the patients' leg: (1) the tibial tuberosity, (2) a point on the tibial crest fifteen centimetres inferior to it, and (3) the medial epicondyle of the femur. A BASELINE (tm) goniometer was then attached to the subjects leg such that diagnostic reference markings were aligned with subjects greater trochanter and lateral malleolus respectively. This allowed the angle of the joint to be monitored constantly. The examiner then manipulated the leg throughout a ROM beginning at $100^{\circ}$ and continuing to $-10^{\circ}$ by $10^{\circ}$ increments. An anterior load of $125 \pm 5$ Newtons was orthogonaly applied to the proximal aspect of the tibia for 
two reasons: (1) the neutral point was difficult to detect clinically and was not considered as being a constant point of evaluation in unloaded conditions, and (2) the deficiency had to be elucidated from bilateral comparisons made between intact knees subject to identical loads. Loading throughout the test protocol remained constant and was continually monitored. This ensured that any variation between instant center graphs generated might be attributed to the phenomenon under study and not to variations which existed in less consistent loading techniques. A pulley mechanism which effectively applied and monitored the desired load throughout the protocol was developed in lieu of a manually applied loading system in which technique was predetermined to be examiner specific and variable. As such, the mechanism designed met three essential criteria: (1) the load was applied orthogonaly to the shank as it moved throughout the range described, (2) the load was applied as a constant force of $125 \pm 5$ newtons regardless of the shanks position in space, and (3) the mechanism accommodated the digitization of three anatomical landmarks as the leg moved throughout the range prescribed. In light of these criteria the following mechanism was designed.

Two steel poles were connected by an articulating joint. One vertical pole was positioned perpendicular to the ground while a shorter horizontal pole was attached orthogonaly to the top of the vertical pole. These poles provided the skeletal frame for the device. Four pulleys were strategically affixed to the poles: two on the vertical pole and two on the horizontal pole. The Stryker force applicator monitored the amount of force applied to the tibia through a spring mechanism and 
corresponding force scale. This device was used in conjunction with the pulleys to monitor the loads generated throughout the procedure. Specifically, a rope passed over each pulley and was attached at one end to the handle of the Stryker. The other end of the rope supported a weighted load of twenty five pounds which hung freely in space. The Stryker was then positioned behind the tibia at the proximal end of the segment such that the application of the prescribed load elicited anterior translation of the tibia at the tibial tuberosity (Fig 4). The pulleys vere positioned such that three anatomical landmarks could be digitized in three unique positions per pulley. Initially these landmarks were digitized at $100^{\circ}, 90^{\circ}$, and $80^{\circ}$ of flexion. Other pulleys facilitated the digitization of the same three anatomical landmarks but with the knee positioned differently in space. For example the second pulley allowed the landmarks to be digitized at $70^{\circ}, 60^{\circ}$, and $50^{\circ}$ respectively. This process was repeated for the remaining two pulleys. In this manner complete digitization of the anatomical landmarks in all positions throughout the range could be accomplished without compromising the established loading criteria. In this way any variation that occurred between instant center profiles generated between knee conditions could then be attributed to the shifting phenomenon of the IC and not to inconsistencies inherent in the loading technique. This procedure was important in the assessment of ligament disruption as the position of the selected anatomical landmarks were effectively described in reference to three orthogonol planes: (1) a transverse plane identified by the $\mathrm{X}$ axis, (2) a frontal plane identified by the $\mathrm{Y}$ axis, and (3) a sagittal plane identified by the $\mathrm{Z}$ axis. These coordinates were ultimately used to plot 
SCHEMATIC OF THE LOADING MECHANISM

DESIGNED TO APPLY AN ORTHOGONOL LOAD

TO THE TIBIA THROUGHOUT A RANGE OF MOTION

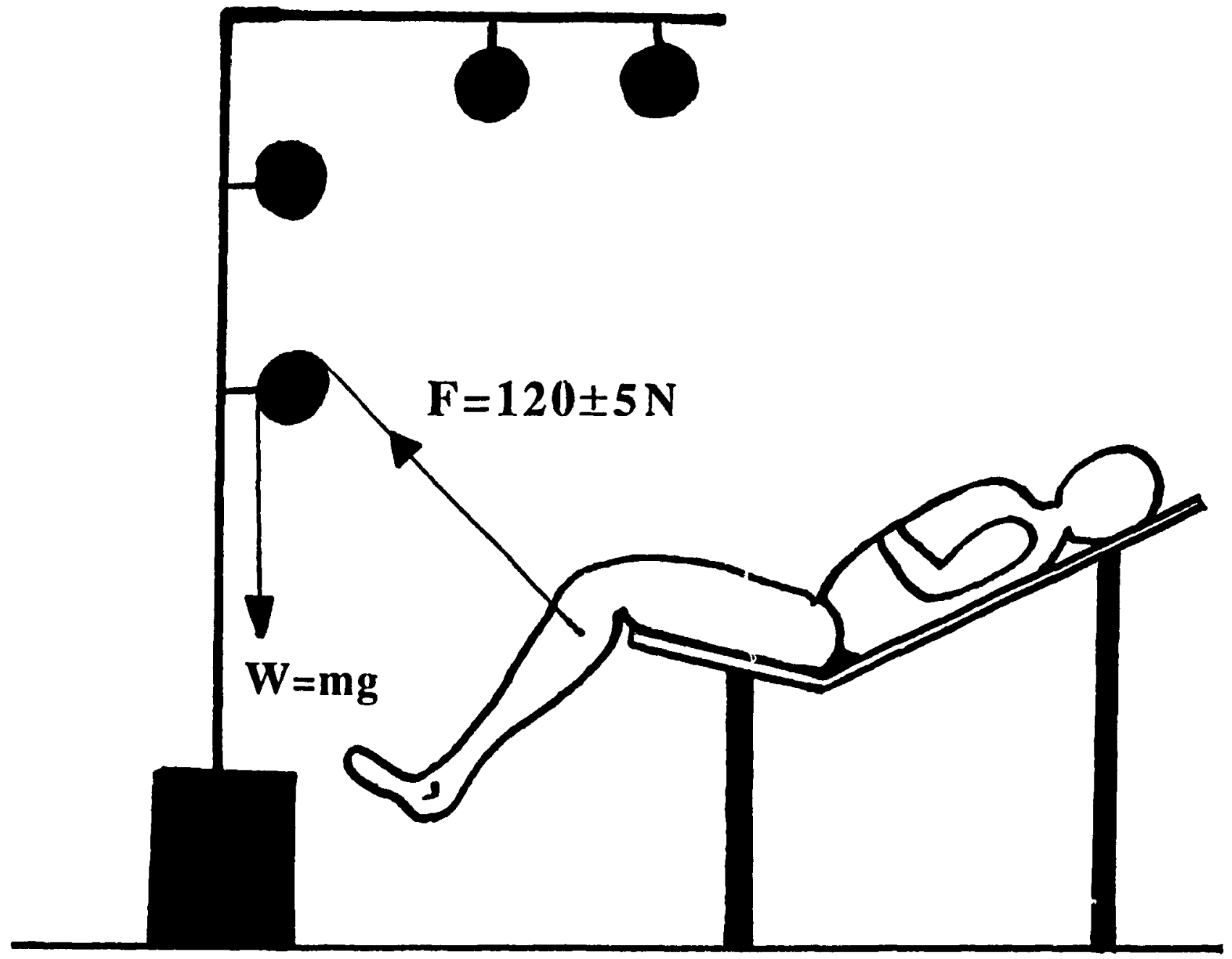

Figure 4 
the instant center graphs according to the method of Reuleaux (1876). They were obtained three sequential times for the intact and involved knees respectively. For the purposes of this investigation only the $Y$ and $\mathrm{Z}$ coordinates were used to compute the displacement of the IC because parameters obtained in the transverse (X) plane in a preliminary study did not seem to characterize the deficiency in specific terms.

\subsection{CALCULATION OF THE INSTANT CENTER}

The instant center coordinates were calculated by a computer program which established the relative position of three anatomical landmarks as they became displaced from one position to another throughout the range. These landmarks included: (1) the tibial tuberosity, (2) a point on the tibial crest approximately fifteen centimetres inferior to it, and (3) the medial epicondyle of the femur. This last point effectively established an origin from which the IC coordinates were measured. In this way, discrepant measures obtained between digitized link coordinates (due to inadvertent shifts of the subjects leg between trials) were compensated for by expressing them relative to an origin that was equally affected by the same motion. The perpendicular bisectors of the the lines connecting corresponding displaced tibial landmarks were then constructed by linear algebraic equations. The intersection of constructed bisectors established the IC whose coordinates were obtained by simultaneously solving the equations of the lines describing the bisectors. This procedure occurred every $10^{\circ}$ throughout the ROM. In this way eleven IC coordinates were 
generated by the coinputer for analysis. Another computer program plotted the IC values. The $\mathrm{Y}$ and $\mathrm{Z}$ coordinate was graphed separately in relation to flexion angle. A spline function established the best fitting curve to connect the plotted coordinates so that the relative displacements of the IC could be quantified in millimetres throughout the entire range. The analysis was restricted to IC coordinates obtained in flexion (between $100^{\circ}$ and $70^{\circ}$ ) and extension (between $30^{\circ}$ and $0^{\circ}$ ). Functional instabilities associated with the deficiency were reported as relative migration displacements throughout the ROM.

\subsection{SIATISTICAL ANALYSIS}

A one-way ANOVA evaluated the statistical significance between knee conditions of the IC of rotation parameters obtained in flexion and extension respectively. Factor $\mathrm{A}$ included two levels of knee condition. Displacement parameters along the $Y$ and $Z$ axis were statistically assessed at the 0.05 level of confidence. Means and standard deviations were also reported for the measures.

\begin{tabular}{|l|c|}
\hline \multicolumn{2}{|c|}{ FACTOR A: KNEE CONDITION } \\
\hline Involved & Intact \\
\hline
\end{tabular}

Additionally, each subjects' joint rotation pattern was evaluated bilateraly and ipsilateraly for shape reliability. Pearson Product 
Moment Coefficients assessed the variability within and between knee conditions.

\begin{tabular}{|c|c|c|}
\cline { 2 - 3 } \multicolumn{1}{c|}{} & RIGHT INVOLVED & LEFT INTACT \\
\hline RIGHT INVOLVED & $\mathbf{a}$ & $\mathbf{B}$ \\
\hline LEFT INTACT & $\mathbf{B}$ & $\mathbf{a}$ \\
\hline
\end{tabular}

NOTE: a denotes ipsilateral "within" knee comparison B denotes contralateral "between" knee comparison

\subsection{SUMMARY}

The ability of clinicians to accurately assess knee joint stability is hampered by the subjectivity associated with proprioceptive examinations. A methodology is proposed to objectively document the functional and kinematic asymmetries observed between involved and intact knees for subjects exhibiting clinical symptoms associated with isolated ACL dysfunction. 


\section{CHAPTER IV}

\section{ANALYSIS AND RESULTS}

\subsection{INTRODUCTION}

The results presented in this chapter are divided into three sections. Section one reflects the preliminary part of the investigation in which the method of Reuleaux (1876) was evaluated for reliability throughout a range of motion (ROM). Three dimensional spatial coordinates used to compute instant center (IC) joint rotation patterns were evaluated for reliability and consistent motion parameters were identified in specific terms at the ends of the range. Section two characterizes the nature and extent of a subjectively diagnosed deficiency. The descriptive statistics presented describe motion parameters obtained by the Genucom Knee Analyzer (GKA) and reflect the structural integrity of the knee joint. Discrepancies in these variables reflect the asymmetrical characteristics between knee conditions for the subject sample investigated. Section three documents the effects of ACL dysfunction on IC joint rotation patterns obtained under a loaded condition and throughout a range of motion. Displacement shifts of IC parameters obtained from joint rotation profiles were thought to reflect the quasi-dynamic link synergy between bone and compliant ligaments (Gerber \& Matter, 1983). 


\subsection{SECTION I}

The reliability of the GKA to accurately quantify inter-trial and within subject variability of the IC of rotation parameter was statistically assessed in late flexion (delta Z) and extension (delta Y). Pearson Product Moment Coefficients (PPMC's) determined the shape reliability of the graphs within trials and between days. These data addressed two important concems: (1) it established the consistency of the IC shifting phenomenon throughout a ROM, and (2) they facilitated a means by which to establish specific and objective motion parameters that accurately reflected link synergy. These data was compiled on a single subject with no previously diagnosed structural knee joint deficiency. Ten trials were performed on two sequential days. The results are presented in Tables $1 \mathrm{~A}$ and $1 \mathrm{~B}$ respectively. No significant differences were observed between and within trials.

Table 1A: Shape Reliability of

IC Rotation Profiles Obtained

Within Trials and Between Days

\begin{tabular}{cccc} 
Day & Trials & $\begin{array}{c}\text { R-Value } \\
\text { Within }\end{array}$ & $\begin{array}{c}(\mathbf{a}=\mathbf{0 . 0 5}) \\
\text { Between }\end{array}$ \\
\hline 1 & $1-10$ & $0.96-0.99$ & \\
2 & $11-20$ & $0.91-0.97$ & \\
1 by 2 & & & $0.94-0.96$
\end{tabular}

Table 1B: Descriptive Statistics;

IC of Rotation Variability ( $\mathrm{mm}$ )

$\begin{array}{cccc} & \text { Day 1 } & \text { Day 2 } & \text { t-value } \\ \text { delta Y } & 3.2 \pm 1.2 & 3.5 \pm 1.4 & t<2.101 \\ \text { delta Z } & 2.6 \pm 1.4 & 2.9 \pm 1.8 & t<2.101\end{array}$




\subsection{SECTION $\amalg$}

It was essential to confirm the nature of a subjectively diagnosed deficiency and to establish the extent of clinical laxity asymmetry between knees for each subject tested for two reasons. Firstly, it provided objective documentation from which to assess the structural specificity of the injury. Secondly, it provided a basis for which to include only those subjects exhibiting statistically significant laxity asymmetries of five millimetres or greater. A series of bilateral laxity tests performed by a certified examiner on the GKA generated the data presented in the following sections.

\subsubsection{SUBJECT INFORMATION}

A total of twenty subjects were initially selected to participate in the investigation. An experienced Orthopaedic Surgeon identified those subjects exhibiting clinical symptoms associated with "isolated" ACL dysfunction by proprioceptive and (whenever possible) arthroscopic means. All subjects were required to fill out a consent form, which described the nature of the study (Appendix $B$ ), and a subject information form (Appendix C). The latter provided the tester with a more detailed description of each subjects pathological history. This information was important to obtain as it supplemented the diagnostic findings of the physician by identifying those patients exhibiting associated structural deficiencies. Furthermore, it provided invaluable information in the iiserpretation of IC of rotation profiles obtained in the latter part of the 
investigation. Because conclusions were to be drawn based on the shapes of the IC patterns generated between knee conditions, it was logical to establish common characteristics of injury between subjects from which shape comparisons would ultimately be made. Relevant information collected from these questionnaires is summarized in Table 2.

Table 2: Subject Information Summary

Subject Gender Age Date of Injury Surgery $A C L$ MM MCL LCL $A C$

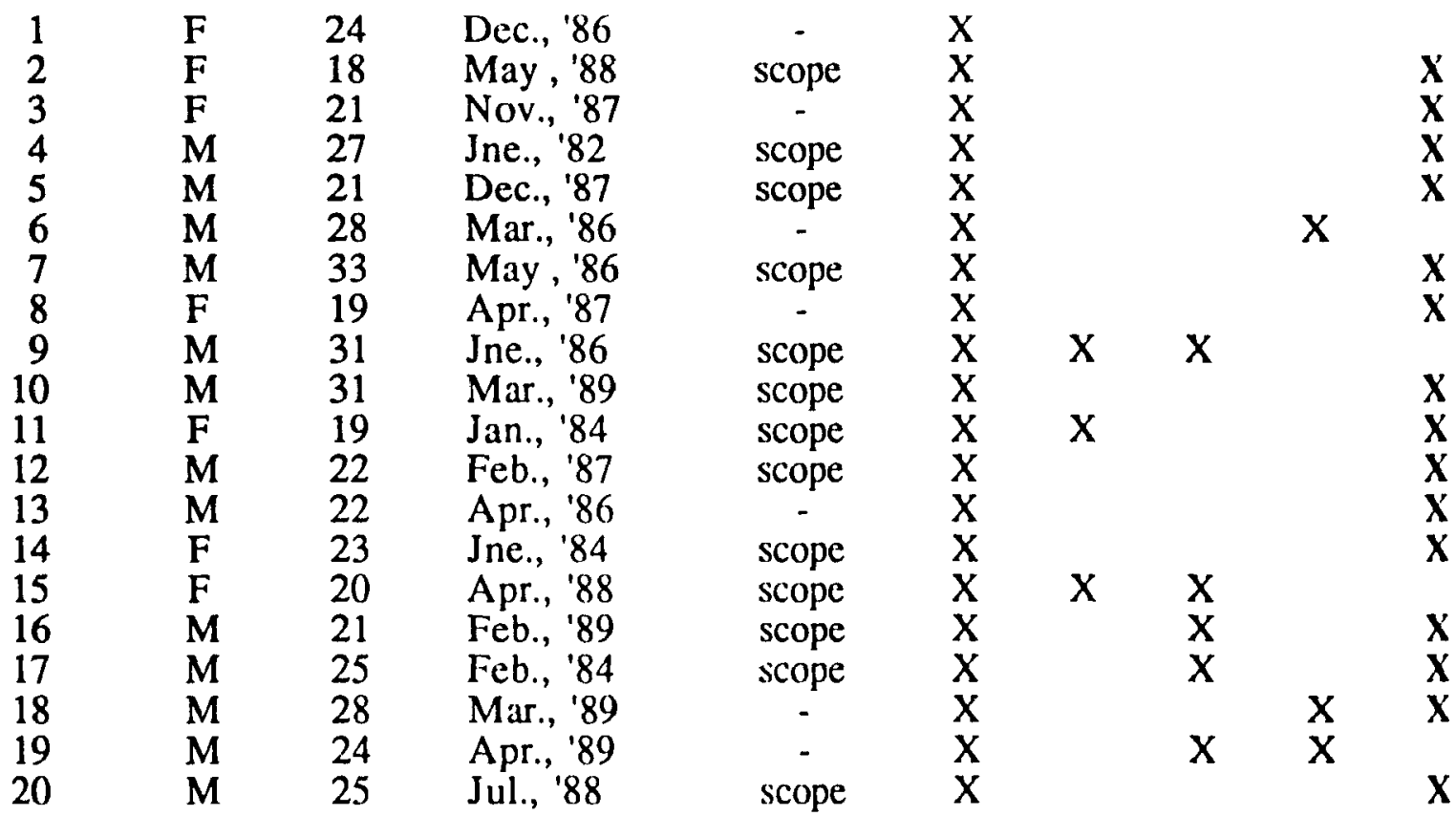

\section{LEGEND}

ACL: Anterior Cruciate Ligament

MM: Medial Meniscus

LCL: Lateral Collate $\cdots a l$ Ligament

* AC: Asymmetry Criteria

The "X's" denote the structures injured for each subject as well as those subjects exhibiting documented laxity asymmetries of five millimetres or 
greater. This "asymmeıry criteria" (AC) reduced the sample size to fifteen subjects. The results presented throughout the remaining pages reflect the data obtained from the reduced sample.

\subsubsection{One-Way ANOVA Results}

One-way ANOVA's evaluated significance between knee conditions on each of the dependent measures identified to characterize the nature and extent of the injury. The results are presented in the tables $3 \mathrm{~A}-3 \mathrm{H}$.

\section{Table 3A: One-Way ANOVA Summary AMRS@20}

\begin{tabular}{|c|c|c|c|}
\hline Source of Variation & $\mathrm{df}$ & F Ratio & F Prob. \\
\hline Between & 1 & 10.96 & $0.0026 *$ \\
\hline Within & 28 & & \\
\hline Total & 29 & & \\
\hline
\end{tabular}

Table 3B: One-Way ANOVA Summary AMRS@30 @

\begin{tabular}{crrr} 
Source of Variation & df & F Ratio & F Prob. \\
\hline Between & 1 & 9.350 & 0.0049 \\
Within & 28 & & \\
Total & 29 & &
\end{tabular}


Table 3C: One-Way ANOVA Summary

AL@90

\begin{tabular}{crcc} 
Source of Variation & df & F Ratio & F Prob. \\
\hline Between & 1 & 0.747 & 0.3947 \\
Within & 28 & & \\
Total & 29 & &
\end{tabular}

Table 3D: One-Way ANOVA Summary AL@ $90^{\circ}$ and $10^{\circ}$ ER

\begin{tabular}{crcc} 
Source of Variation & df & F Ratio & F Prob. \\
\hline Between & 1 & 1.638 & 0.2111 \\
Within & 28 & & \\
Total & 29 & &
\end{tabular}

Table 3E: One-Way ANOVA Summary AL@30

\begin{tabular}{crrr} 
Source of Variation & df & F Ratio & F Prob. \\
\hline Between & 1 & 9.985 & $\mathbf{0 . 0 0 3 8}$ \\
Within & 28 & & \\
Total & 29 & &
\end{tabular}

Table 3F: One-Way ANOVA Summary

AL@20

Source of Variation df F Ratio F Prob.

$\begin{array}{lrrr}\text { Between } & 1 & 22.93 & 0.0000 \\ \text { Within } & 28 & & \\ \text { Total } & 29 & & \end{array}$




\section{Table 3G: One-Way ANOVA Summary \\ ITR@90}

\begin{tabular}{crcc} 
Source of Variation & df & F Ratio & F Prob. \\
\hline Between & 1 & 2.750 & 0.1055 \\
Within & 28 & & \\
Total & 29 & &
\end{tabular}

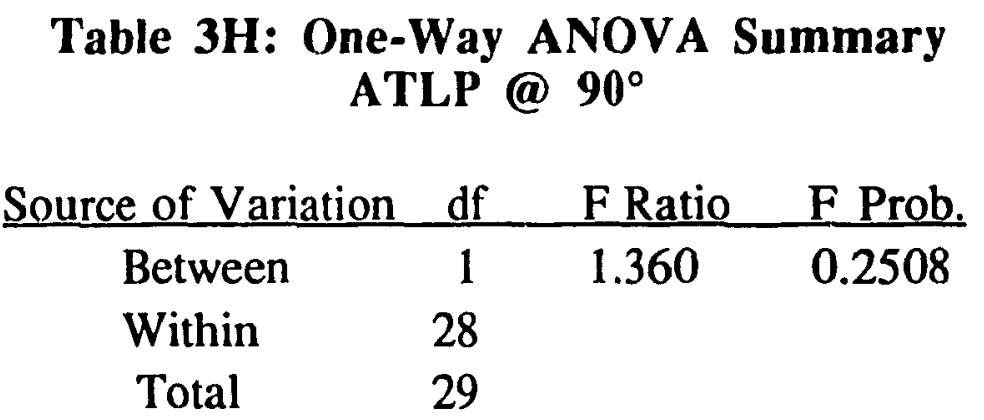

\subsubsection{DESCRIPTIVE STATISTICS}

The means and standard deviations for each dependent measure are summarized in the tables $4 \mathrm{~A}$ through $4 \mathrm{E}$. A number of "conditions" are identified in each table. These conditions identify the angle at which each of the dependent measures were evaluated and are clearly defined. The dependent measures of interest included: anterior terminal laxity (AL), anterior midrange stiffness (AMRS), internal tibial rotation (ITR), and translation of the medial (ATMP) and lateral (ATLP) tibial plateau. 
CONDITIONS:

Condition 1: $20^{\circ}$

Condition 2. $30^{\circ}$

Condition 3: $90^{\circ}$

Condition 4: $90^{\circ}$ with $10^{\circ} \mathrm{ER}$

Condition 5: $90^{\circ}$ with $10^{\circ}$ IR

Table 4A: Descriptive Statistics;

Anterior Laxity in $\mathbf{m m}$

Condition Intact Involved

F Prob.

1

$6.8 \pm 2.2 \quad 12.9 \pm 4.5$

$0.0000 *$

2

$10.9 \pm 3.4$

$15.7 \pm 4.8$

$0.0038 *$

3

$8.8 \pm 2.7 \quad 10.2 \pm 3.4$

0.2184

4

$7.1 \pm 2.5 \quad 8.8 \pm 4.6$

0.2111

Table 4B: Descriptive Statistics;

Anterior Midrange Stiffness in $\mathbf{N} / \mathbf{m m}$

Condition

1

2

3

4
Intact

$17.8 \pm 6.7$

$10.7 \pm 3.2$

$15.1 \pm 8.2$

$23.0 \pm 11.4$
Involved

$10.7 \pm 6.3$

$7.3 \pm 2.9$

$12.8 \pm 5.9$

$16.2 \pm 6.0$
F Prob.

$0.0030 *$

0.0049 *

0.3947

$0.5000^{*}$ 
Table 4C: Descriptive Statistics;

Internal Tibial Rotation in degrees

$\begin{array}{clcc}\text { Condition } & \text { Intact } & \text { Involved } & \text { F Prob. } \\ 3 & 5.3 \pm 2.8 & 6.7 \pm 2.3 & 0.1050 \\ 3 & 21.1 \pm 5.5 & 21.8 \pm 8.4 & 0.7413\end{array}$

Table 4D: Descriptive Statistics;

Anterior Translation of the

Medial Tibial Plateau in $\mathbf{m m}$

$\begin{array}{cccc}\text { Condition } & \text { Intact } & \text { Involved } & \text { F Prob. } \\ 4 & 0.6 \pm 1.1 & 0.6 \pm 1.1 & 0.9448\end{array}$

Table 4E: Descriptive Statistics;

Anterior Translation of the

Lateral Tibial Plateau in $\mathbf{m m}$

$\begin{array}{cccc}\text { Condition } & \text { Intact } & \text { Involved } & \text { F Prob. } \\ 5 & 0.9 \pm 1.2 & 1.7 \pm 2.6 & 0.2508\end{array}$

\subsubsection{Two-Factor ANOVA Summary Table}

Table 5 summarizes the results obtained by the two-factor ANOVA. Anterior midrange stiffness (AMRS) and anterior terminal laxity (AL) were evaluated at each flexion angle across knee condition. Asterisks indicate Main Effects at the 0.05 level of confidence. 


\section{Table 5: Two-Factor ANOVA Summary of Results}

\begin{tabular}{|c|c|c|c|c|}
\hline FACTOR B & \multicolumn{4}{|c|}{ FACTOR A: FLEXION ANGLE } \\
\hline DEPENDENT MEASURE & $20^{\circ}$ & $30^{\circ}$ & $90^{\circ}$ & $90^{\circ} \& 10^{\circ}$ ER \\
\hline AMRS & $*$ & $*$ & & $*$ \\
\hline AL & $*$ & $*$ & & \\
\hline
\end{tabular}

\subsubsection{Tukey Post-Hoc Mean Comparisons}

A Tukey Post-Hoc comparison identified the locus of the significance for each dependent measure obtained across flexion angle. Table 6 presents the results expressed as interactions between the means presented in Tables $4 A$ and $4 B$ respectively.

\section{Table 6: Tukey Post-Hoc Mean Comparisons}

\begin{tabular}{lcccc} 
Condition & Variable & Mean Comparisons & Tukey Range \\
INTACT & \multirow{2}{*}{ AL (mm) } & (1) 10.9 with 6.8 & 3.74 \\
& & (2) 10.9 with 7.1 & 3.74 \\
& \multirow{2}{*}{ AMRS (N/mm) } & (1) 23.0 with 10.7 & 3.74 \\
INVOLVED & \multirow{2}{*}{ AL (mm) } & (2) 23.0 with 15.1 & 3.74 \\
& & (1) 15.7 with 4.8 & 3.74 \\
& & (2) 15.7 with 8.8 & 3.74 \\
& \multirow{2}{*}{ AMRS (N/mm) } & (1) 16.2 with 10.7 & 3.74 \\
& & (2) 16.2 with 7.3 & 3.74 \\
& & (3) 12.8 with 7.3 & 3.74
\end{tabular}




\subsection{SECTION III}

Section III documents the effects of ACL dysfunction on the IC of joint rotation profiles obtained under a loaded condition and throughout a ROM. Intra-subject reliability coefficients established the statistical characteristics of the displacement patterns ipsilateraly and contralateraly.

\subsubsection{INTRA-SUB.JECT SHAPE RELIABULITY}

Each subjects' joint rotation pattern was elucidated three times according to the method of Reuleaux (1876). Three dimensional spatial coordinates obtained on the GKA were used to plot the desired rotation patterns. Each pattern was evaluated contralateraly and ipsilateraly for shape reliability throughout a ROM. Pearson Product Moment Coefficients determined the variability within and between knee conditions. The results are presented in Table 7. 


\section{Table 7: Intra-Subject Reliability Coefficients Within and Between Knee Conditions}

\begin{tabular}{crcc} 
& \multicolumn{2}{c}{ WITHIN } & BETWEEN \\
Subject & Intact & Involved & \\
2 & 0.69 & 0.61 & 0.28 \\
3 & 0.68 & 0.75 & 0.59 \\
4 & 0.83 & 0.74 & 0.31 \\
5 & 0.85 & 0.67 & 0.26 \\
7 & 0.86 & 0.84 & 0.56 \\
8 & 0.73 & 0.81 & 0.20 \\
10 & 0.93 & 0.71 & 0.55 \\
11 & 0.92 & 0.63 & 0.19 \\
12 & 0.64 & 0.47 & 0.89 \\
13 & 0.97 & 0.80 & 0.69 \\
14 & 0.78 & 0.64 & 0.13 \\
16 & 0.85 & 0.97 & 0.82 \\
17 & 0.85 & 0.97 & 0.82 \\
18 & 0.88 & 0.68 & 0.53 \\
20 & $\underline{0.79}$ & 0.82 & 0.71 \\
& & & \\
& $\mathbf{0 . 7 9}$ & $\mathbf{0 . 7 2}$ & $\mathbf{0 . 3 8}$
\end{tabular}

\subsubsection{ONE-WAY ANOVA RESULTS}

One-way ANOVA's determined the statistical significance of the IC of rotation parameters between knee conditions. These parameters were evaluated in late flexion (delta $\mathrm{Z}$ ) and in extension (delta $\mathrm{Y}$ ). The results are presented in tables 8 and 9 respectively. 
Table 8: One-Way ANOVA Summary of the IC of Rotation Parameter Obtained Between $100^{\circ}$ and $70^{\circ}$

\begin{tabular}{lrrr} 
Source of Variation & df & F Ratio & F Prob. \\
\hline Between & 1 & 0.7344 & 0.3993 \\
Within & 28 & & \\
Total & 29 & &
\end{tabular}

Table 9: One-Way ANOVA Summary of IC of Rotation Paramcters Obtained Between $30^{\circ}$ and $-10^{\circ}$

\begin{tabular}{lrcc} 
Source of Variation & df & F Ratio & F Prob. \\
\hline Between & 1 & 7.021 & 0.0130 \\
Within & 28 & & \\
Total & 29 & &
\end{tabular}

\subsubsection{DESCRIPTIVE STATISTICS}

The means and standard deviations were determined for delta $\mathrm{Z}$ and delta $\mathrm{Y}$ across knee condition. The results are presented in Table 10.

Table 10: Descriptive Statistics of the Instant Center Parameter (mm)

\begin{tabular}{cccc}
\multicolumn{4}{c}{ Knee Condition } \\
Variable & Intact & Involved & F Prob. \\
\hline delta Z & $3.05 \pm 1.42$ & $3.59 \pm 1.87$ & 0.3993 \\
delta $Y$ & $5.07 \pm 1.86$ & $6.97 \pm 2.06$ & 0.0131
\end{tabular} *




\subsubsection{DISPLACEMENT PROFILE $\triangle$ SSESSMENT}

Figures 5 and 6 depict the displacement profiles of the $\mathrm{IC}$ of rotation as it moved throughout a ROM. The vertical (Y) axis reflects the amount of anterior and posterior translation of the IC of rotation measured relative (o) a fixed femur. The non-linear displacement profiles depict a series of "peaks" and "valleys" which appear at specific points throughout the range. These curves have been interpreted along three specific sections of the profile and are identified as three distinct phases: (1) a preliminary phase (between $100^{\circ}$ and $70^{\circ}$ ), an intermediate phase (between $70^{\circ}$ and $30^{\circ}$ ), and (3) a tertiary phase (between $30^{\circ}$ and $0^{\circ}$ ). These phases appear to contain consistent patterns of displacement throughout the range and reflect the quasi-dynamic link synergy for intact and involved knees. Specifically, posterior shifting of the IC of rotation was documented in the preliminary phase for all profiles generated. The shape and magnitude of this migration proved to be a consistent motion characteristic regardless of the subjects' knee condition. The peaks and valleys documented in the intermediate region of the curve (between $70^{\circ}$ and $30^{\circ}$ ) demonstrated less consistent trends. Although this phase of the displacement profile was characterized by a peak occurring between $45^{\circ}$ and $30^{\circ}$ (preceded by a valley between $70^{\circ}$ and $45^{\circ}$ ), the relative magnitude of "inflection points" defining these curves varied within and between subjects. Tertiary phases revealed consistent profile patterns within subjects but the magnitude of inflection points defining the peaks and valleys varied considerably botween knee conditions. Interestingly this phase did not seem to teriniinate as a definitive peak or valley within or between knee conditions. 


\section{INSTANTANEOUS CENTER OF ROTATION \\ PROFILE OF AN INVOLVED KNEE \\ ALONG A VERTICAL (Y) AXIS \\ THROUGHOUT' A RANGE OF MOTION}



Figure 5 
INSTANTANEOUS CENTER OF ROTATION

PROFILE OF AN INTACT KNEE

ALONG A VERTICAL (Y) AXIS

THROUGHOUT A RANGE OF MO'TION

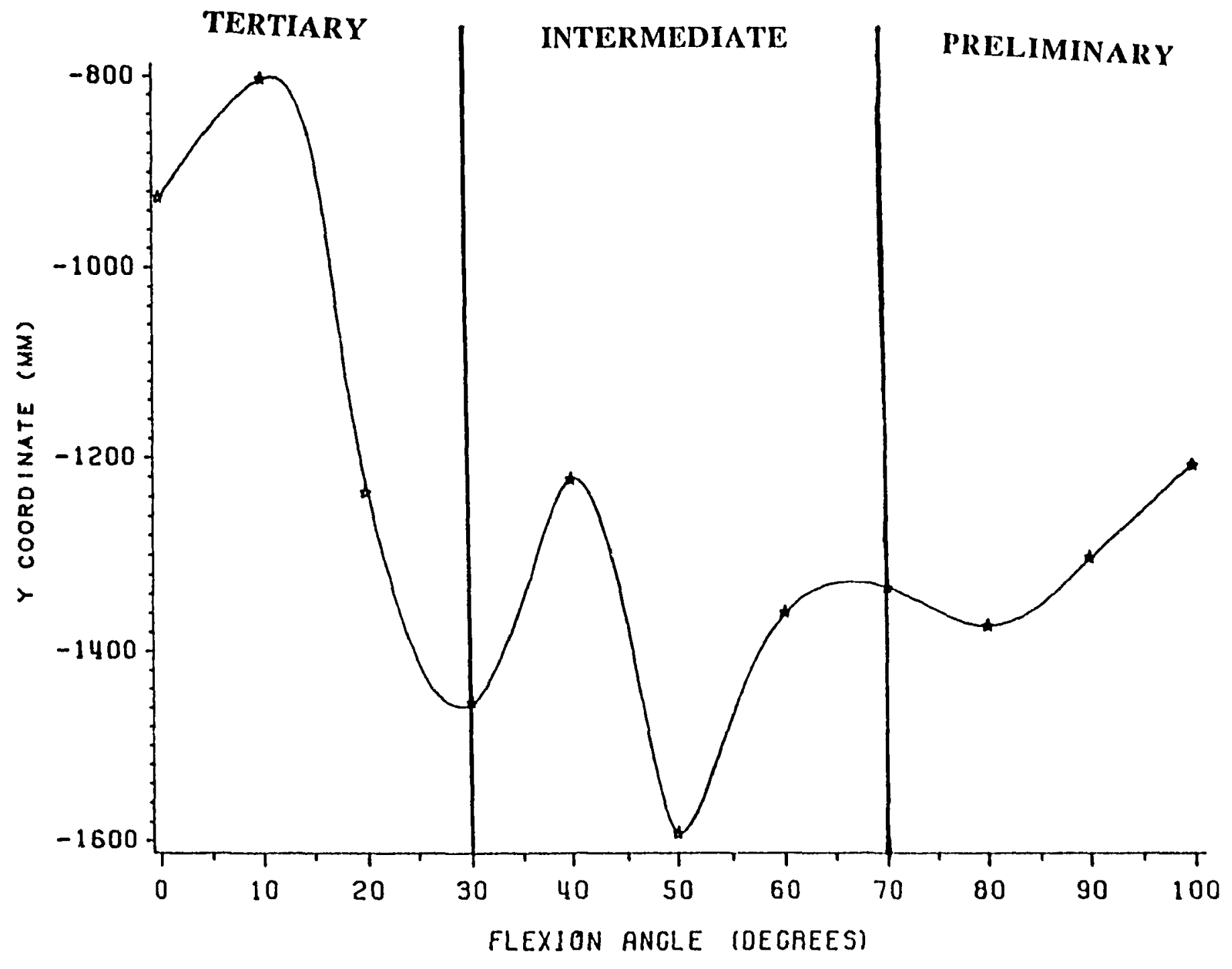

Figure 6 
Figures 7 and 8 depict displacement profiles of the IC of rotation parameters quantified along the sagittal $(\mathrm{Z})$ axis. Migration of the IC along this axis reflected proximal and distal translation of the IC relative to a fixed femur. These profiles were interpreted in three phases which identified specific ranges of knee flexion throughout a ROM. Non-linear displacement profiles revealed a series of peaks and valleys within each phase. $A$ consistent distal shifting of the IC of rotation characterized all profiles obtained between and within knee conditions. The shape, magnitude, and number of inflection points comprising these profiles appeared to be symmetrical. 


\section{INSTANTANEOUS CENTER OF RO'TA'TION \\ PROFILE OF AN INVOLVED KNEL \\ ALONG A SAGITTAL (Z) AXIS \\ THROUGHOUT A RANGE OF MO'TION}

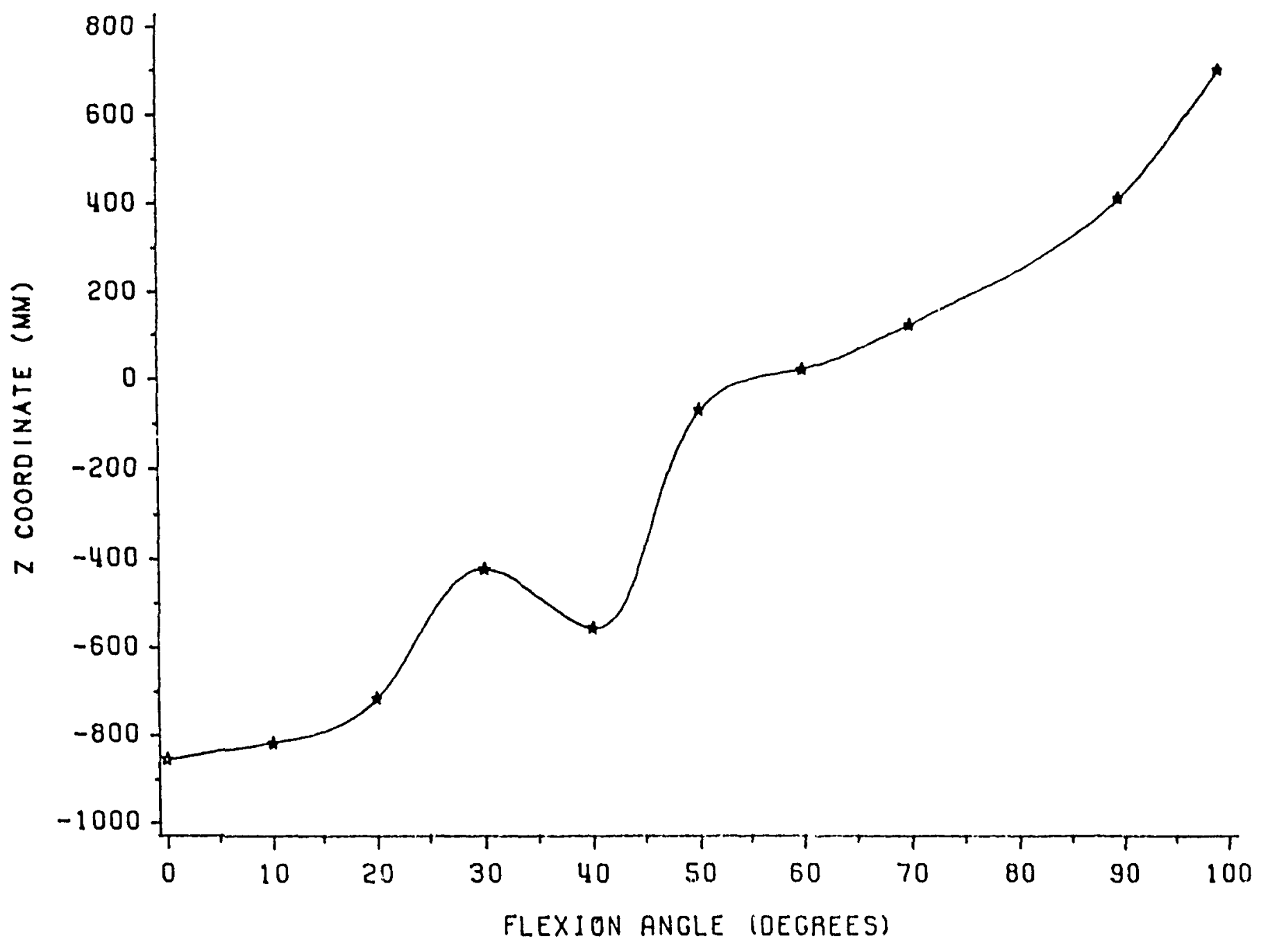

Figure 7 
INSTAN'TANEOUS CENTER OF ROTATION PROFILE OF AN INTACT KNEE

ALONG A SAGITTAL (Z) AXIS

THROUGHOUT A RANGE OF MOTION

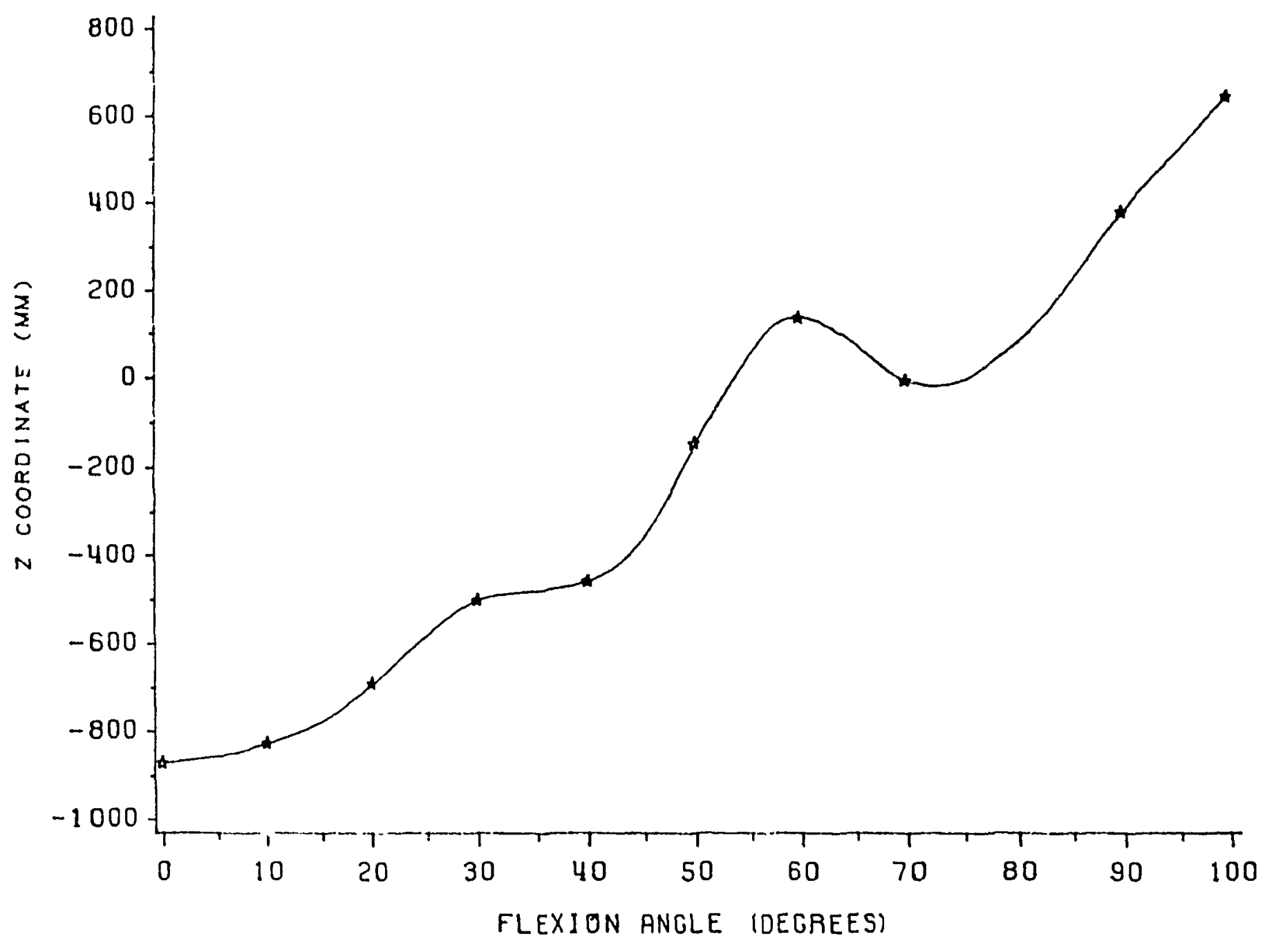

Figure 8 


\section{CHAPTER V}

\section{DISCUSSION OF RESULTS}

\subsection{INTRODUCTION}

Proprioceptive methodologies for assessing knee joint stability often result in discrepant diagnoses (Markolf et al., 1976). This stems from the subjective interpretation of clinical evaluations. The purpose of this investigation was to employ a knee arthrometer as an objective testing device to characterize instant center (IC) joint rotation patterns of $\mathrm{ACL}$ deficient knee joints. It was hypothesized that, in conjunction with three dimensional motion parameters obtained by the same device, unique kinematic profiles reflecting in-vivo joint integrity could ultimately be established to characterize the deficiency in specific terms. This facilitated the need to implement a protocol which quantified "uni" and "multi-planar" dependent measures.

The data presented in this chapter are divided into three sections which reflect the component parts of the investigation. Section one evaluates the reliability of the technique used to compute IC of rotation motion parameters. Sectior two examines the nature and extent of a subjectively diagnosed deficiency. Section three documents joint rotation profiles which were obtained under a loaded condition and throughout a ROM. These profiles reflect the quasi-dynamic link synergy between bone and compliant ligaments (Gerber \& Matter, 1983; Frankel et al. 1971). 


\subsection{SECTION I}

Pearson Product Moment Coefficients (PPMC's) determined the shape reliability of the IC of rotation profiles within trials and between days. These data were essential to obtain as they established the consistency of the GKA to accurately define three dimensional spatial coordinates. Table 1A presents the results. PPMC's obtained within trials on day one varied from 0.96 to 0.99 and on day two from 0.91 to 0.94 . Between-day coefficients ranged from 0.94 to 0.97 . Two conclusions can be drawn from these data. Foremost, it is clear that IC of rotation coordinates computed according to the method of Reuleaux (1876) provided a stable measure of joint motion throughout a range. This supported the reliability of the technique in documenting link synergy. Secondly, since three dimensional spatial coordinates were used to compute two dimensional displacement profiles, and since good shape reliability within the between profiles was observed, the reliability of the GKA to quantify orthogonol knee motion was confirmed.

Table $1 \mathrm{~B}$ documents $\mathrm{IC}$ of rotation variability at the ends of the range. Migratory shifts of the IC in flexion (delta $\mathrm{Z}$ ) were $2.6 \pm 1.4 \mathrm{~mm}$ for day 1 and $2.9 \pm 1.88 \mathrm{~mm}$ for day 2 . In extension (delta $Y$ ) these shifts were observed to be $3.5 \pm 1.4 \mathrm{~mm}$ and $3.2 \pm 1.2 \mathrm{~mm}$ between days. Mean parameters were not found to be significantly different between days when assessed by a two-tailed t-test at the 0.05 confidence level. These data confirmed the presence of a consistent and quantifiable shifting phenomenon of the IC throughout a ROM. 


\subsection{SECTION U}

Motion characteristics were established between knee conditions to determine the nature and extent of a subjectively diagnosed deficiency. The results of bilateral clinical tests performed on the GKA by a certified examiner were summarized in tables $4 \mathrm{~A}$ through $4 \mathrm{E}$. These parameters were interpreted in light of the secondary research hypotheses initially proposed in chapter one. Specifically these hypotheses pertained to the relationship between stiffness and laxity measures and the angle at which they were quantified.

\subsubsection{LAXITY MEASURES}

The data in Table 4A document significant laxity asymmetries between knee conditions for measures quantified in extension (conditions $1 \& 2$ ) but not for laxity measures quantified in flexion (conditions $3 \& 4$ ). Clearly, ACL dysfunction does not de-stabilize the joint uniformly throughout a ROM. There are two logical explanations for this. The first relates to the functional characteristics of the ligament. Since the ACL is comprised of two principle parts - an anteromedial band (AMB) and a posterolateral band (PLB) - and since each band contributes to different aspects of knee, stability throughout a ROM, it follows that the band component resisting knee extension was the only structure of the ACL significantly damaged. King et al., (1986) and Girgis et al., (1975) establised that the bulk of the PLB was tightest between $30^{\circ}$ and $0^{\circ}$ degrees and was the principle structure resisting hyper-extension. The same study also established that the AMB only became tight in flexion (between $100^{\circ}$ and $90^{\circ}$ degrces). 
The authors suggested that the clinically perceptible drawer sign would occur in flexion under two conditions: (1) when the ACL was entirely severed, or (2) when the AMB of the ACL was structurally deficient. Although the subjects participating in this investigation were diagnosed as being ACL deficient, no information was obtained regarding the specific location of the injury along the ACL. However all subjects were asked to describe the circumstances surrounding their injury and most of them reported a hyper-extension type mechanism. As the PLB of the ACL is tightest in extension (while the AMB remains loose by comparison), it is not unreasonable to support a band-specific explanation for the laxity measures obtained with the knee positioned at $20^{\circ}$ and $30^{\circ}$ degrees.

The second explanation for the results obtained in Table 4A relates to the structural characteristics of the knee joint in flexion. Rossenberg \& Rausmussen (1984) suggested that a knee positioned at $90^{\circ}$ flexion functions like a "roller and trough". The authors likened the acutely convex structure of the posterio femoral condyles to a "roller" and the tibial articular surfaces (with intact menisci) to a "trough". They proposed that forward movement of the tibia on the femur during the drawer test is prohibited without "deflecting" the joint space vertically to accommodate the roller-trough geometry of the articulating bones (Fig 9). Under normal circumstances this defleciion is prevented by the tense capsule and collateral ligaments that contribute to joint stability. Consequently the ACL is effectively "buffered" by the roller-trough system. During the Lachmans test however, the anterior contact point of the femur assumes a "flatter" orientation on the tibia due to the nature of the contacing surfaces 
79)

SCHEMATIC DEPICTING THE

"ROLLER-TROUGH" MECHANISM IN FLEXION

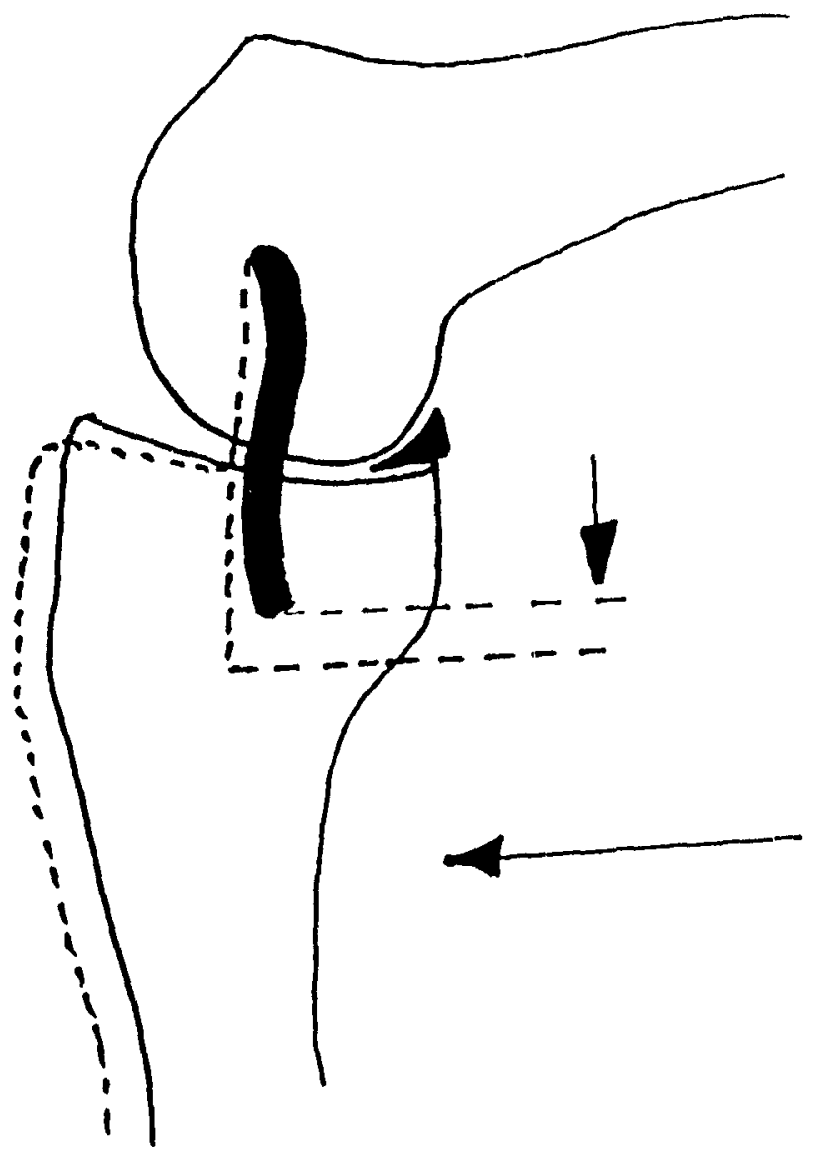

Figure 9 
between the distal femoral condyles and the tibial plateaus in extension (Fig 10). This geometrial relationship promotes anterior translation of the tibia on the femur without requiring a perquisite vertical deflection.

The results of Tukey Post-Hoc Mean Comparisons for laxity and stiffness measures obtained in the various "conditions" are presented in Table 6. Although the literature is replete with studies documenting the effectiveness of the Lachmans test in identifying ACL dysfunction, controversial information exists regarding the specific angle at which the test should be performed to elicit optimal results. (Typically the knee is positioned somewhere between thirty and fifteen degrees.) Interactions of mean laxity parameters across flexion angle addressed secondary hypothesis one. It stated: there will be no significant differences between AL parameters evaluated...by the Lachmans test performed at $30^{\circ}$ and $20^{\circ}$ flexion for intact and involved knees. Analysis of the data requires that this hypothesis be rejected. A significant difference was found to exsist between laxity measures at each flexion angle. Specifically AL measures quantified at $30^{\circ}$ were found to be significantly higher than $\mathrm{AL}$ measures quantified with the knee positioned in any other angle. The clinical iniplication of this finding is the assumption that, to be considered useful descriptors of knee motion, anterior laxity data should be performed at $30^{\circ}$ on the GKA.

\subsubsection{STIFFNESS MEASURES}

The data in Table 4B document stiffness asymmetries. Significant differences were noted between knee conditions 1, 2, and 4. Interaction of AMRS parameter means across flexion angle addressed secondary 


\section{SCHEMATIC DEPICTING THE}

"ROLLER-TROUGH" MECHANISM

IN EXTENSION

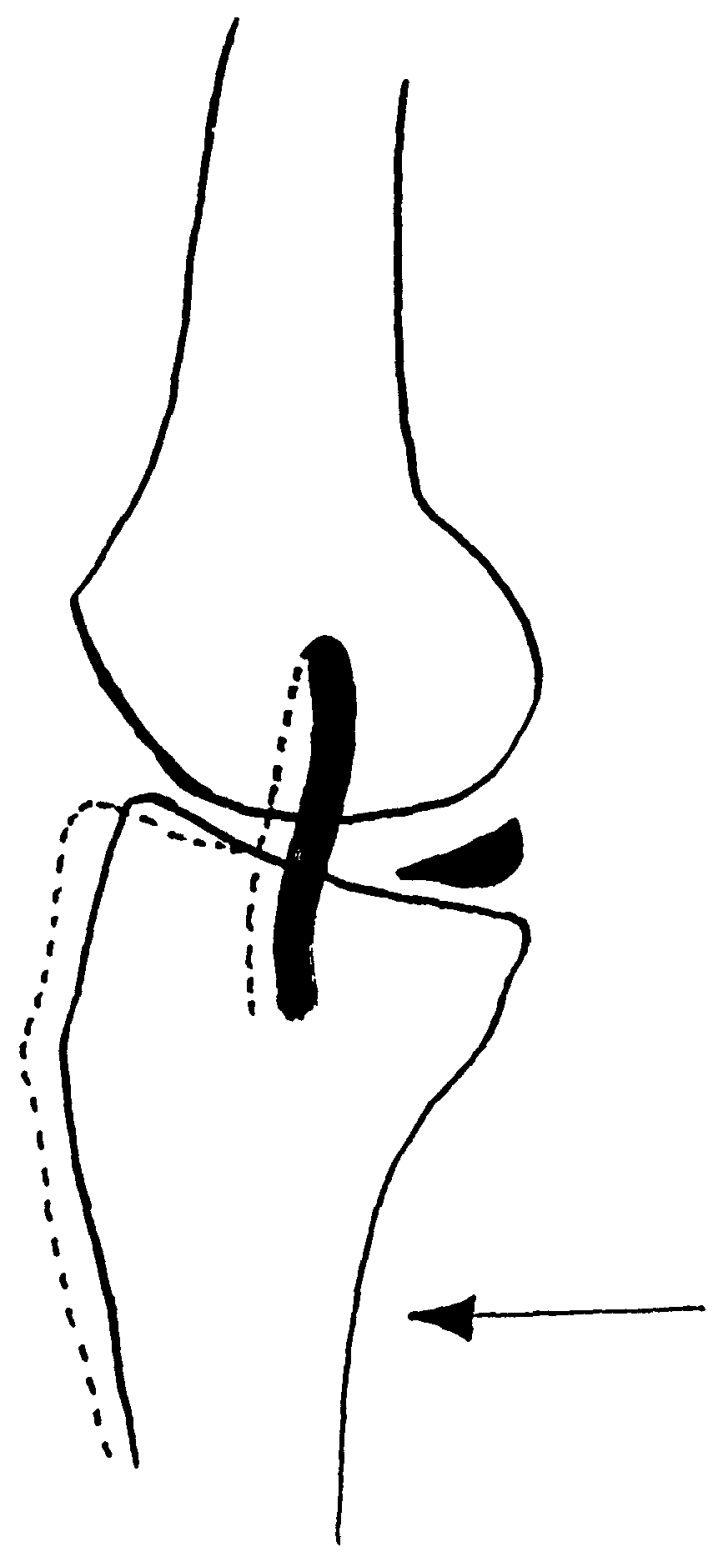

Figure 10 
hypothesis two. It stated: there will be no significant difference between AMRS parameters... while performing the Lachmans test at $30^{\circ}$ and $20^{\circ}$ flexion for intact and involved knees. Analysis of this data reveals significant stiffness asymmetries to exist between values quantified midrange in extension (conditions 1 and 2) and in flexion (condition 4). These results are not surprising. Since AMRS is computed as the inverse slope of force-displacement data obtained from laxity curves, and since laxity measures were greatest in extension for involved knees, it is logical to expect stiffness measures to follow a similar trend. Unlike laxity results however, a significant difference in stiffness asymmetry was noted in condition 4. Tukey Post-Hoc comparisons established this position $\left(90^{\circ}\right.$ flexion with $10^{\circ}$ external rotation) as being a potential "tell-tale" sign of joint integrity because significant stiffness asymmetries were documented between this condition and all others. However, caution must be exercised in interpreting the clinical importance of this finding. Two factors must be considered. Foremost, performing an anterior drawer in which the tibia is externally rotated potentially results in compression of the joint capsule. Furthermore, intact meniscal pads block the motion occurring between the femoral condyles and the tibial plateaus. As $80 \%$ of subjects tested in this investigation had no meniscal injuries associated with the primary deficiency, the possibility of inflated stiffness values (due to meniscal blocking of the condyles) is ever present. Secondly, torsional loads applied at the joint are frequently perceived by subjects as being uncomfortable, unnatural, or even painful. Frequently "defense mechanisms" kick-in during a clinical evaluation. Inadvertent contraction of supporting musculature by the subjects in the hopes of accommodating the "unnatural" motion being elucidated is not unlikely. Johnson \& Hull (1988) determined 
that resisting musculature could increase joint stiffness by as much as $320 \%$. Inflated stiffness values affect joint stability by decreasing potentially significant laxity measures (Markolf et al.,1978).

\subsubsection{TORSIONAL INSTABILITIES}

Tables $4 \mathrm{C}$ through $4 \mathrm{E}$ document the rotational characteristics associated with isolated ACL dysfunction. These data address secondary research hypotheses three and four. Surprisingly, no significant differences were observed for any of the conditions investigated. This may be explained by considering the concept of secondary restraints. Noyes et al., (1980) suggested that joint laxity is not only affected by the loss of a single ligament. but also to a change in the interaction between a deficient ligament and supporting seconday structures. These structures provide secondary restraints to knee motion once the primary restraint has been structurally compromised. Consequently, the results of clinical tests might well elicit deflated laxity measures despite the deficit present in the primary restraining ligament. Although $47 \%$ of the subjects tested in this investigation reported damage to other structures, the dara presented in this study do not suggest secondary structures were significantly compromised.

\subsection{SECTION III}

The third part of the investigation documented the $\mathrm{IC}$ of joint rotation profiles of knee joints according to the method of Reuleaux (1876). The patterns were obtained three times bilateraly under a loaded condition and throughout a ROM. 


\subsubsection{INTRA-SUB.JECT RELIABILITY OF IC PROEULES}

Each subjects' IC of joint rotation profile was evaluated ipsilateraly and contralaterly for shape reliability. The results presented in Table 7 document low Pearson Product Moment Coefficients (PPMC's) for joint rotation patterns obtained within and between knee conditions for the subject sample tested, when compared to PPMC's obtained on a single subject in Part one of the investigation. Profile coefficients evaluated ipsilateraly ranged from 0.59 to 0.88 for intact knees and from 0.47 to 0.97 for involved knees. Mean coefficients were determined to be 0.79 and 0.72 for intact and involved knees respectively. These results suggest that ipsilateral shape reliability between profiles generated within knee conditions are relatively stable. Furthermore, mean coefficients demonstrate that the shape reliability of joint rotation patterns are compromised to the same degree within knee conditions. When compared to PPMC's obtained for a single subject, shape reliability for the sample decreased $19 \%$ for intact knees and $24 \%$ for involved knees. This observation might be explained by considering the "stress" each joint was subjected to during the evaluation procedure. Anterior loads of $120 \pm 5$ Newtons were applied throughout a ROM while spatial coordinates were quantified accordingly. This procedure was performed three times per knee for each subject tested. Each trial tooh approximately 10 minutes to complete. In light of this, it is not unreasonable to expcct inadvertent contraction of supporting musculature to manifest at some point throughout the protocol in an attempt by the subject to alleviate a potentially stressful situation. In the single subject case, 10 trials were performed over a series 
of hours. Consequently the joint was not subjected to the same intense testing protocol experienced by the other subjects. Since IC of rotation profiles are influenced by the compliant properties of ligaments and the geometry of articulating bone, and since active contraction of musculature is documented to affect the interaction between these structures, it is likely that IC profiles depicting "pure" link synergy became compromised by the effects of inadvertent muscular contraction of subjects being tested.

Shape reliability coefficients decreased by $60 \%$ for contralateral comparisons obtained for the subject sample when compared to reliability coefficients obtained for the single subject. This observation is easily explained. Contralateral comparisons for knees in which no deficiency has been documented should be reflected by similar IC profiles. The data presented in this study support this notion. PPMC's obtained between knee conditions for a single subject without a documented deficiency displayed good shape reliability ranging from 0.94 to 0.96 . However, contralateral profile coefficients obtained for the ACL deficient subjects revealed poor shape reliability ranging from 0.13 to 0.89 . (The mean coefficient was determined to be 0.38 ). Two conclusions can be drawn from this: joint synergy is influenced by the structural integrity of the $A C L$, and (2) this synergy can be quantified by applying the method of Reuleaux (1876) .

\subsubsection{DISPLACEMENT PROFILE ASSESSMENT}

Figures 5 and 6 depict the displacement profiles of the IC of rotation as it moves throughout a ROM. The vcrtical (Y) axis documents the amount of anterior and posterior translation of the IC measured relative to a fixed 
femur. (A.lthough IC of rotation proililes were generated for every subject, only the results of two subjects appear in this study because they reflected the trends and characteristics observed in the rotation profiles).

The non-linear curves were interpreted along three specific sections of the profile but only the preliminary and tertiary phases were considered as being important in characterizing knee motion. In the preliminary phase a demonstrated posterior shift of the IC was consistently observed between $100^{\circ}$ and $70^{\circ}$ degrees flexion (Fig 5). The net displacement of the IC within this range was demonstrated to be insignificant between knee conditions. There are two explanations for this phenomenon. With the knee positioned between $100^{\circ}$ and $70^{\circ}$ degrees flexion, displacement shifts quantified by a vertical plane in response to a sagittaly applied load will not manifest to the extent they might if quantified in a sagittal plane. Secondly, the effect of gravity acting through the joint can not be overlooked. The subject was positioned in the GKA such that the flexed knee hung freely in space. Clearly this situation does not mirror normal weight bearing conditions. In this position, the posterior shift of the IC in flexion might well be effected more by gravitational forces and less by synergistic link mechanisms. Furthermore the ACL prevents tibial translations along a sagittal plane and not along a vertical plane. As a result, even deficient ACL's will not effect significant displacement shifts within the confines of a plane that masks the effects of its true motion. It was for this reason that IC profiles were also quantified along a $\mathrm{Z}$ axis throughout the $\mathrm{ROM}$ - to account for inherent motion limitations of uni-pianar analysis. 
The patterns which occurred in the intermediate phase (between $70^{\circ}$ and $30^{\circ}$ ) demonstrated good shape reliability contralateraly, however, data were not extrapolated from this part of the curve. Since the focus of the investigation was to examine the effects of the primary restraining ligament, ard since secondary restraints engage midrange to promote joint stability, the intermediate phase was considered inconsequential in light of the hypotheses established at the on-set of the experiment.

Tertiary analysis of profiles obtained between knee conditions demonstrated significant displacement asymmetries of the IC of rotation. This region was characterized in all instances by a series of anterior and posterior migrations of the IC reflecting corresponding "peaks and valleys". Although the shapes of these profiles did not seem to characterize the deficiency in specific terms, the relative magnitude between inflection points defining them did. Specifically, IC displaceement shifts for intact knees revealed a "net" anterior translation of $5.7 \pm 1.86 \mathrm{~mm}$ in comparison with $6.97 \pm 206 \mathrm{~mm}$ for involved knees. These were determined to be significantly different at the 0.05 level of confidence. Since profiles were computed from IC coordinates which defined the limits of anterior and posterior translation throughout a ROM, and since reliability coefficients were demonstrated to be low between these coordinates (Table 7), it was clear that kinematic asymmetries between knee conditions would be demonstrated by discrepancies in the relative magnitudes of the inflection coordinates and not by discrepancies in the actual shapes of the profiles obtained. A qualitative analysis of the profiles confirmed this observation. Comparisons between intact and involved displacement migrations of the IC along a vertical axis reveals each profile to contain four "valleys" at 
identical points throughout the range (Figs 7 and 8). Visually, profiles may be distinguished from each other by a "peak" present at $0^{\circ}$ for the involved knee which is noticeably absent from the intact condition. Interestingly, this terminal peak appeared quite randomly throughout the profiles generated; it was just as likely to manifest in intact knee profiles as it was for involved.

Figures 7 and 8 depict displacement profiles of the IC of rotation along the sagittal $(Z)$ axis. These curves were also interpreted along three distinct phases. Qualitative analysis of the curves reveal consistent distal shifting of the IC of rotation as the knee approaches extension. Quantitatively displacement parameters were evaluated in the preliminary phase only. (Analysis of the profiles along a sagittal axis would account for motion characteristics associated with joint synergy in flexion). Results document a $3.05 \pm 1.42 \mathrm{~mm}$ distal translation of the IC for intact knees in comparison with $3.59 \pm 1.87 \mathrm{~mm}$ shift for involved knees. This shift was not determined to be significant at the 0.05 level of confidence and suggests that pathological changes in knee joint dynamics occur primarily in extension.

\subsubsection{CLINICAL IMPLICATIONS}

The results of the joint rotation profiles obtained bilateraly suggest that flexion and extension do not proceed identically for joints presented with structural deficits when compared to normal knees. A specific pattern of abnormality was demonstrated to exist in extension. This pattern was reflected by displacement migrations of the IC of rotation between $30^{\circ}$ and 
$0^{\circ}$ and not by shape discrepancies between profile curves throughout a ROM. Clearly the ACL does not de-stabilize the joint uniformly. All the data presented in this study suggest that knee motion is compromised greatest in extension for subjects exhibiting clinical symptoms associated with ACL dysfunction. For individuals wanting to participate in sports on a recreational or competitive level, it is essential that the joint be stabilized by some mechanism in extension. Knee orthoses have been developed with this restraining property in mind. Many commercially available braces are designed to prohibit hyperextension by mechanically restricting the ROM.

\subsection{SUMMARY}

In the past rotation patterns have been documented objectively by roentenographic methodologies in which a. series of $\mathrm{x}$-rays were taken of the knee as it moved throughout a ROM. Researchers determined that joint deficiencies were characterized by the shapes of the profiles obiained. Gerber \& Matter (1983) and Frankel et al., (1971) demonstrated that normal IC motion was characterized by parallel movement of the joint surfaces which reflected gliding. Abnormal knee motions in the same study were characterized by anterior and distal shifts of the IC from $40^{\circ}$ to $20^{\circ}$ and reflected joint compression and distraction.

The IC of rotation profiles obtained in this study suggest that the rolling-gliding mechanism is a complex interactive phenomenon which is not effectively characterized by profile shape. Although deficient knees displayed a pattern similar to the one observed by other investigators, these patterns did not differ significantly from patterns computed for intact 
knees. More accurately, IC of rotation patterns obtained by the method of Reuleaux (1876) reflected the extent by which joint mechanics had been compromised because of the deficiency and not the kinematic locus of the injury itself. 


\section{CHAPTER VI}

\section{SUMMARY AND CONCLUSIONS}

\section{LINTRODUCTION}

Injuries to the knee joint have received more attention in the medical literature over the past ten years than any other joint in the body (Clancy, 1983). In light of the increasing incidence of knee injuries, an emphasis has been placed on the importance of early clinical recognition of joint symptoms (Girgis et al., 1975). In the past, ident sication of knee deficits were limited to a physicians' ability to elicit joint symptoms accompanying ligament dysfunction, however, such proprioceptive methodologies are subjective. As such, clinicians and researchers have recognized the need to provide objective data which would assist them in decision making regarding knee joint stability. The purpose of this investigation therefore, was to develop a protocol that would employ an objective testing device in an attempt to characterize knee motion in specific terms.

\subsection{RESULTS AND FINDINGS}

\subsubsection{THREE DIMENSIONAL MOTION CHARACTERISTICS}

Laxity and stiffness characteristics were quantified by the Genucom Knee Analyzer (GKA) in a variety of positions. These parameters reflected the three dimensional motion characteristics of the knee joint and 
were obtained in reference to three orthogonol planes. Anterior terminal laxity (AL) was measured in four conditions. Each condition reflected a different angle at which the variables were obtained. The highest reported laxity value was $15.7 \pm 4.8 \mathrm{~mm}$ for an involved knee positioned at $30^{\circ}$. However, anterior laxity asymmetries between knee conditions were greatest at $20^{\circ}$ with a $6.1 \mathrm{~mm}$ difference observed bilateraly. This compared with a $4.8 \mathrm{~mm}$ bilateral laxity asymmetry observed between knee conditions at $30^{\circ}$. Similar trends were noted for stiffness (AMRS) parameters quantified at identical flexion angles. These data suggest that functional properties of the ACL are compromised most in extension.

The rotational characteristics of the $\mathrm{ACL}$ were established by quantifying tibial plateau displacement in a transverse plane. Interestingly, there appeared to be no significant differences between knee conditions for the subjects tested in the study. This finding refutes some research indicating that structural damage to the ACL results in increased rotatory instability (Furman et al., 1976; Roy \& Wang, 1987; Markolf et al., 1984). This observation is explained by considering the concept of secondary restraints. Secondary restraining structures "engage" when the joint is stressed to promote knee stability (Noyes et al., 1980). Furthermore, some of these investigations examined the rotational characteristics of the knee joint in-vitro. Clearly these results negate the interactive co:tinuity that supporting musculature, ligaments, and the joint capsule have in maintaining joint integrity by their invasive design. The clinical implication of these findings is that knee joint stability is not compromised uniformly throughout a ROM. 


\subsubsection{PROFLE ASSESSMENT OF THE IC OF ROTATION}

IC of rotation profiles were obtained according to the method of Reuleaux (1876) under a loaded condition and throughout a ROM. The non-linear displacement curves were evaluated along three specific sections of the profile. Tertiary phase analysis of profiles obtained between knee conditions demonstrated significant displacement asymmetries of the IC in extension (delta $Y$ ) but not in flexion (delta Z). Although the shapes of these profiles did not seem to characterize the deficiency in specific terms, the relative magnitude between inflection points defining them did. This suggests that the rolling - gliding mechanism is a complex interactive synergy between articulating bony geometry and compliant ligaments. This synergy must be evaluated as an integrated mechanism which cannot be resolved into its component kinematic parts.

\subsection{SUMMARY}

This investigation attempted to document specific motion characteristics associated with ACL deficits. The laxity and stiffness parameters associated with the clinical evaluation have been well established in the literature. However, the quasi-dynamic interaction between bone and compliant ligaments throughout a ROM have not been studied to the same extent. Results presented in this study suggest that the method of Reuleaux (1876) has a demonstrated potential for characterizing link synergy and is reflected by IC of joint rotation profiles. Abnormal displacement migrations were demonstrated to occur in extension. These abnormalities 
correlated well with clinical findings. Migration displacements evaluated between $30^{\circ}$ and $0^{\circ}$ in the vertical (Y) axis varied from $2.41 \mathrm{~mm}$ to 10.43 $\mathrm{mm}$ for intact knees and $3.76 \mathrm{~mm}$ to $11.03 \mathrm{~mm}$ for deficient knees. Mean parameters were quantified as being $5.07 \pm 1.86 \mathrm{~mm}$ and $6.97 \pm 2.06 \mathrm{~mm}$ for intact and involved knees respectively. Using a "cut-off" point of 6.0 $\mathrm{mm}$, the methodology proposed in this stludy identified $83 \%$ of the subjects tested as being ACL deficient. Although $17 \%$ of the subjects would have been mis-diagnosed according to this criteria, it must be remembered that IC profiles provide integrated joint information in a "quasi-dynamic" situation as opposed to conventional measures obtained under more "static" conditions. Furthermore, IC data was quantified in the hopes of supplementing clinical measures; not in the hopes of replacing them.

\subsection{CONCLUSIONS}

The following conclusions may be drawn from the results presented in this investigation: (1) that ACL dysfunction does not de-stabilize the joint uniformly throughout a ROM, (2) that the rnethod of Reuleaux (1876) is a technique that demonstrated effectiveness in establishing profile asymmetries between knee conditions along a vertical ( $\mathrm{Y}$ ) axis, and (3) that abnormalities in IC of rotation profiles are attributed to discrepancies in the relative magnitude between inflection points defining them and not to the shapes of the profiles themselves. 


\subsection{RECOMMENDA TIONS FOR FURTHER STUDY.}

This investigation supports the notion that IC of rotation motion parameters may prove effective in the design and evaluation of knee orthoses. Since IC profiles are conipromised greatest in extension, and since a quantitative measure of normal link synergy can be obtained within the limits of this range, the ability of the device to promote "normal" synergistic mechanisms might be objectively evaluated. 


\section{REFERENCES}

Ahmed, A. M., A. Hayden. D.L., Burke, \& K.H. Chan. (1987). In-vitro ligament tension pattern in the flexed knee in passive loading. Joumal of Othopaedic Research, 5 (2), 217-230.

Arnoczky, S. P. (1983). Anatomy of the anterior cruciate ligament. Clinical Orthopaedic and Related Research, 172, 19-25.

Brien, H., \& Hoshizaki, T.B. (1986). A comparison of mechanical knee laxity testers and the lachmans test in anterior cruciate deficient knees. Unpublished manuscript.

Butler, D.L., Noyes, F.R., \& Grood, E.S. (1980). Ligamentous restraints to anterior-posterior drawer in the liuman knee. The Journal of Bone and Joint Surgery, 62 (2), 259-270.

Cabaud, H.E. (1983). Biomechanics of the anterior cruciate ligament. Clinical Orthopaedics and Related Research, 172, 26-31.

Clancy, W.G., Jr. (1983). Knee ligamentous injury in sports: the past, present and future. Medicine and Science in Sports and Exercise 15 (1), 9-14.

Crownsinshield, R., Popoe, M.H., \& Johnson, R.J. (1976). An analytical model of the knee. Journal of Biomechanics, 9, 397-405.

Edixhoven, P., Huiskes, R., de Graff, R., van Rens, T.J. (1987). Accuracy and reproducibility of instrumented knee-drawer tests. Journal of Orthopaedic Research, 5 (.3), 378-387.

Frankel, V.H., Burstein, A.H., \& Brooks, D.B. (1971). Biomechanics of internal derangement of the knee. The Journal of Bone and Joint Surgery, 53-A (5), 945-962.

Frankel, V.H. \& Nordin, M. (1980). Basic biomechanics of the skeletal system. Philadelphia: Lea \& Febieger. 
Furman, J.L., Marshall, D.V.M., \& Girgis, F.G. (1976). The anterior cruciate ligament: a functional analysis based on postmortem studies. The Journal of Bone and Joint Surgery, 58-A (2), 179-185.

Fukubayashi, T., Torzilli, P.A., Sherman, M.F., \& Warren, R.F. (1982). An in-vitro biomechanical evaluation of anterior-posterior motion of the knee. The Joumal of Bone and Bone and Joint Surgery 64-A (2), 258-264.

Gerber, C., \& Matter, P. (1983). Biomechanical analysis of the knee after rupture of the anterior cruciate ligament and its primary repair. The British Journal of Bone and Joint Surgery, 65-B (4), 391-399.

Girgis, F.G., Marshall, J.L., \& Al Monajem, A.R.S. (1975). The cruciate ligaments of the knee joint. Clinical Orthopaedics and Related Research, 106, 216-231.

Gurtler, R.A., Stine, R., \& Torg, J.S. (1987). Lachman test evaluated. Clinical Orthopaedic and Related Research, 216, 141-150.

Harding, M.L., \& Blakemore, M.E. (1980). The instant center pathway as a parameter of joint motion-an experimental investigation of a method of assessment of knee ligament injury and repair. Engineering in Medicine 2 (4), 195-200.

Hoshizaki, T.B., \& Matthews, S. (1989). Intertester and day to day variation of knee joint laxity characteristics as measured by the genucom. Unpublished manuscript.

Houghston, J.C., Andrews, J.R., Cross, M.J., \& Moschi, A. (1976). Classification of knee ligament instabilities. The Journal of Bone and Joint Surgery, 58-A (2), 173-179.

Hsieh H.H., \& Walker, P.S. (1976). Stabilizing mechanisms of the loaded and unloaded knee joint. The Journal of Bone and Joint Surgery, 58-A (1), 87-93.

Huiskes, R.E., de Graaf, R., Van Rens, J.G., \& Slooff, T.J. (1987). Accuracy and reproducibility of instrumented knee drawer tests. Joumal of Orthopaedic Research 5 (3), 378-387. 
Johnson, R.J. (1983). The anterior cruciate ligament problem. Clinical Orthopaedic and Related Research, 172, 14-18.

Johnson C., \& Hull, M.L. (1988). Parameter identification of the human lower limb under dynamic, transient torsional loading. Joumal of Biomechanics, 21 (5), 401-415.

Kennedy, J.C., \& Fowler, P.J. (1971). Medial and anterior instability of the knee. The Journal of Bone and Joint Surgery 53-A (7), 12571270 .

Kennedy, J.C., Weinberg, H.W., \& Wilson, A.S. (1974). The anatomy and function of the anterior cruciate ligament. The Joumal of Bone and Joint Surgery, 56-A (2), 223-234.

Kennedy, J.C., Hawkins, R.J., \& Willis, R.B. (1977). Strain gauge analysis of knee ligaments. Clinical Orthopaedics and Related Research, 129, 225-229.

King, S., Butterwick, D.J., \& Cuerrier, J.P. (1986). The anterior cruciate ligament: a review of recent concepts. The Journal of Orthopaedic and Sports Pyhsical Thearapy 8 (3), 110-122.

Lipke, J.M., Janecki, C.T., Nelson, C.L., McLeod, M.S., Thompson, C., Thompson, J., \& Haynes, D.W. (1981). The role of incompetence of the anterior cruciate and lateral ligaments in anterolateral and anteromedial instability. The Journal of Bone and Joint Surgery, 63-A. 242-253.

Louie, J.K., \& Mote, C.D. (1987). Contribution of the musculature to rotatory and torsional stiffness at the knee. Journal of Biomechanics, 20 (3), 281-300.

McLeod, W. (1985). The biomechanics and function of the secondary restraints to the anterior cruciate ligament. Orthopaedic Clinics of North America, 16 (2), 165-169.

Markolf, K.L., Mensch, J.S., \& Amstutz, H.C. (1976). Stiffness and laxity of the knee-the contribution of the supporting structures. The Journal of Bone and Joint Surgery, 58-A (5), 583-593. 
Markolf, K.L., Graff-Radford, A., \& Amustutz, H.C. (1978). In-vivo knee stabilty. The Journal of Bone and Joint Surgery 60-A (5), 664674.

Markolf, K.L., Kochan, A., \& Amstutz, H.C. (1984). Measurement of knee stiffness and laxity in patients with documented absence of the anterior cruciate ligament. The Journal of Bone and Joint Surgery, 66A (2), 242-253.

Markolf, K.L., \& Amstutz, H.C. (1987). The clinical relevance of instrumented testing for ACL insufficiency. Clinical Orthopaedic and Related Research, 223, 198-207.

Marshall, J.L., Baugher, W.H. (1980). Stability examination of the knee: a simple anatomic approach. Clinical Orthopaedics and Related Research, 146, 78-85.

Marquette, S,H. (1988). Stabilizing anterior cruciate ligament injuries: biomechanical requirements of orthotic design. Orthotics and Prosthetics, 41 (4), 18-28.

Moeinzadeh. M.H., Engin, A.E., \& Akkas, N. (1983). Two-dimensional dynamic modelling of the human knee joint. The Journal of Biomechanics, 16 (4), 253-264.

Müeller, W. (1985). The knee: Form, function and ligament construction. New York: Springer-Verlay.

Müeller, W., Biedert, R., Hefti, F., Jakob, R.P., Munzinger, V., \& Staubli, H.U. (1988). OAK: a new way to assess knee ligament injuries. Clinical Orthopaedics and Related Research, 232, 37-50.

Noyes, F.R., Grood, E.S., Butler, D.L., \& Malek, M. (1980). Clinical laxity tests and functional stability of the knee and related biomechanical concepts. Clinical Orthopaedic and Related Research, 146, 84-89.

Noyes, F.R., Pekka, A.M., Matthews, D.S., \& Butler, D.L. (1983). The symptomatic anterior cruciate deficient knee. The Joumal of Bone and Joint Surgery, 65-A (2), 203-211. 
Odensten, M. \& Gillquist, J. (1985). Functional anatomy of the anterior cruciate ligament and a rationaie for reconstruction. The Journal of Bone and Joint Surgery, 67-A (2), 257-265.

Oliver, J.H. \& Coughlin, L.P. (1987). Objective knee evaluation using the genucom knee analysis system. The American Journal of Sports Medicine, $15(6), 571-578$.

Reuleaux, F., (1876). The kinematics of machinery: outline of a theory of machines. MacMillan, London

Roy, G., \& Wang, Y. (1987). The role of cruciate ligaments in maintaing knee joint stability. Clinical Orthopaedics and Related Research, 215, 65-71.

Rossenberg, T.D., \& Rasmussen, G.L. (1984). The function of the anterior cruciate ligament during anterior drawer and lachmans testing. The American Joumal of Sports Medicine, 12 (4), 318-322.

Rovere, G.D., \& Adair, D.M. (1983). Anterior cruciate deficient knees: a review of the literature. The Ameican Journal of Sports Medicine, 11 (6), 412-417.

Shoemaker, S.C., \& Markolf, K.M. (1985). Effects of joint load on the stiffness and laxity of ligament deficient knees. The Joumal of Bone and Joint Surgery, 67-A (1), 136-146.

Soudan, K., \& Van Audekercke, R. (1979). Methods, difficulties and inaccuracies in the study of human joint kinematics and pathokinematics by the instant axis concept. Joumal of Biomechanics, 12, 27-33.

Solomonow, M., Baratta, R., Zhou, B.H., Shoji, H., D'Ambrosia, R. (1986). Histological update and new developments on the EMG-force relationships of skeletal muscle. Orthopaedics, 2 (11), 1541-1543.

Solomonow, M., Baratta, R., Zhou, B.H., Shoji, H., Bose, W., Beck, C., D'Ambrosia, R. (1987). The synergistic action of the anterior cruciate ligament and thigh muscles in maintaing joint stability. American Journal of Sports Medicine, 15 (3), 207-213. 
Torzilli, P. A., Greenberg, R.L., \& Insall, J. (1981). An in-vivo biomechanical evaluation of the anterior-posterior motion of the knee. The Journal of Bone and Joint Surgery, 63-A (6), 960-968.

Walker, P.S., Shoji, H., \& Erkman, M.J. (1972). The rotational axis of the knee and its significance to prosthesis design. Clinical Orthopaedics and Related Research, 89, 160-170.

Welsh, R.P. (1980). Knee joint structure and function. Clinical Orthopaedics and Related Research, 147, 7-13. 


\section{APPENDIX A \\ CLINICAL GRADING SCALE}

The classification scale described by Houghston et al., (1976) dealing with rotatory and straight knee ligament instabilities utilized a grading system for the severity of the instability. This system was proposed by the Committee on the Medical Aspects of Sports of the American Medical Association and was published (1968) in a handbook entitled "Standard Nomenclature of Athletic Injuries".

The grading scale was as follows:

Grade 1+: Mild instability (0-5mm)

Grade 2+: Moderate instability $(5-10 \mathrm{~mm})$

Grade 3+: Severe instability (10-15 mm)

For the purposes of this investigation the scale was modified as follows:

Grade 0 No apparent tibio-femoral displacement

Grade 1 Displacement between 0-4 mm

Grade 2 Displacement between 5-9 mm

Grade 3 Displacement between 10-15 mm

Grade 4 Displacement greater than $15 \mathrm{~mm}$

This was a subjective value decided upon by the examining Orthopedic Surgeon while performing the Lachmans test. It was later confirmed by arthroscopic examination whenever possible. 


\section{APPENDIX B}

\section{Informed Consent}

The studies you have agreed to participate in are designed to evaluate anterior cruciate ligament (ACL) deficiencies. There are three distinct parts representing three distinct studies. Two studies are specifically designed to evaluate functional knee braces. The third study is designed to evaluate the biomechanical characteristics of ACL dysfunction. (You may be participating in one, two, or all of the knee studies but this information will be communicated to you at the on-set of testing).

The Genucom Knee Analyzer (GKA) is a non-invasive research tool which will be used to gather a portion of the data required for analysis. During the Genucom assessment, various forces will be applied to both knee joints by a certified examiner in a series of clinical tests. A maximum force of $33 \mathrm{lbs}$. $(110 \mathrm{~N})$ will be applied to the joint. Additionally, the thigh muscle will be restrained by the device. The other non-invasive testing device to be used in this study is the CYBEX. This isokinetic machine will be used to measure the amount of force generated by the thigh muscle while the brace is positioned on the knee. In addition you will be asked to demonstrate two functional activities: jumping and kicking. These activities will be filmed.

It is important to appreciate that any one or series of these proposed tests may cause some minor discomfort to you. Therefore your participaticn in this study can be discontinued at your discretion at ANY time throughout the protocol by communicating your intention to the technician. As such, you may refuse to complete one or all of the proposed tests.

All results obtained in this study become the property of MzGill Biomechanics Laboratory. Confidentiality will be respected for all subjects involved in the study. The results and the interpretation of the results will be made available to you upon request at the completion of the study.

I have read and understand this form. My signature below reflects my consent to be a participant in this ('hese) study (studies).

Name (Print) Date

Signature Telephone 
APPENDIX C

Biomechanics of Sport

McGil! University

Subject Information Summary

Date:

Gender:

Name:

D.O.B.: $\mathrm{m}$

d

y

Address:

Height;

Weight:

Phone: (h)

Physician:

(w)

Type of Injury: LEFT; ACL PCL MCL LCL MM LM Other RIGHT; ACL PCL MCL LCL MM LM Other Previous Surgery:

Ligament Replacement: Goretex Dachron Other

Mechanism of Injury:

Date of Injury:

Other Comments: 